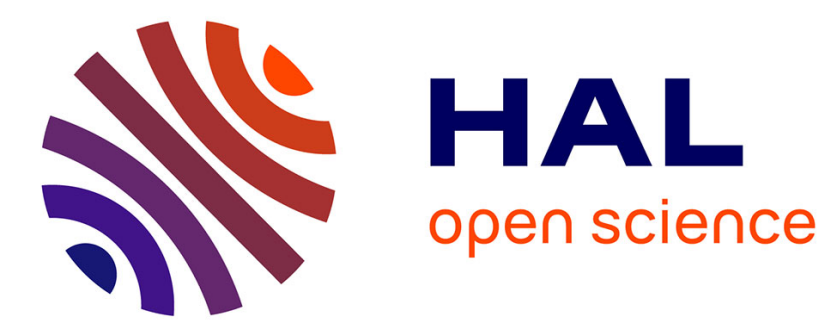

\title{
On the explicit reconstruction of a Riemann surface from its Dirichlet-Neumann operator
}

\author{
Gennadi Henkin, Vincent Michel
}

\section{To cite this version:}

Gennadi Henkin, Vincent Michel. On the explicit reconstruction of a Riemann surface from its Dirichlet-Neumann operator. 2005. hal-00004910v6

\section{HAL Id: hal-00004910 https://hal.science/hal-00004910v6}

Preprint submitted on 3 Oct 2005

HAL is a multi-disciplinary open access archive for the deposit and dissemination of scientific research documents, whether they are published or not. The documents may come from teaching and research institutions in France or abroad, or from public or private research centers.
L'archive ouverte pluridisciplinaire HAL, est destinée au dépôt et à la diffusion de documents scientifiques de niveau recherche, publiés ou non, émanant des établissements d'enseignement et de recherche français ou étrangers, des laboratoires publics ou privés. 


\title{
ON THE EXPLICIT RECONSTRUCTION OF A RIEMANN SURFACE FROM ITS DIRICHLET-NEUMANN OPERATOR
}

\author{
GENNADI HENKIN AND VINCENT MICHEL
}

\begin{abstract}
This article gives a complex analysis lighting on the problem which consists in restoring a bordered connected riemaniann surface from its boundary and its Dirichlet-Neumann operator. The three aspects of this problem, unicity, reconstruction and characterization are approached.
\end{abstract}

\section{Contents}

1. Statements of the main results 1

2. Intrinsic EIT on Riemann surfaces 11

3. Unicity under existence assumption 14

\begin{tabular}{ll}
3.1. & Proof of theorem 1. \\
\hline
\end{tabular}

$\begin{array}{lll}\text { 4. Existence and reconstruction, proof of theorem 2 } & 17\end{array}$

5. Proofs of characterizations theorem 3a, 3b and $3 \mathrm{~d} \quad 19$

5.1. Proof of A.theorem 3a 20

5.2. Proof of B.theorem 3a 21

5.3. Proof of C.theorem 3a 22

5.4. Proof of A.theorem 30 23

5.5. Proof of B.theorem 30 24

5.6. Proof of theorem 3b. $\quad 27$

6. Characterizations, effective or affine 27

6.1. Explicit integro-differential characterization 27

\begin{tabular}{lll}
\hline $6.2 . \quad$ Affine characterization & 32 \\
\hline
\end{tabular}

$\begin{array}{ll}\text { References } & 32\end{array}$

\section{Statements of the MAin RESUlts}

Let $\mathcal{X}$ be an open bordered riemannian real surface (i.e. the interior of an oriented riemannian two dimensional real manifold whose all components have non trivial one dimensional smooth boundary) and $g$ its metric. Using the boundary control method, Belishev and Kurylev [4] 6 have started the study of the inverse problem consisting in recovering $(\mathcal{X}, g)$ from the operators $N_{\lambda}: C^{\infty}(b \mathcal{X}) \ni u \mapsto\left(\partial \widetilde{u}_{\lambda} / \partial \nu\right)_{b \mathcal{X}}$ where $b \mathcal{X}$ is the boundary of $\mathcal{X}, \nu$ is the normal exterior unit to $b \mathcal{X}$ and $\widetilde{u}_{\lambda}$ is the unique solution of $\Delta_{g} U=\lambda U$ such that $\left.U\right|_{b \mathcal{X}}=u$. The principal result of [6] implies that the knowledge of $\lambda \mapsto N_{\lambda}$ on an non empty open set of $\mathbb{R}_{+}$determines $(\mathcal{X}, g)$

Date: October 3, 2005.

1991 Mathematics Subject Classification. Primary 58J32, 32C25; Secondary 35R30 . 
up to isometry. The important question to know if $(\mathcal{X}, g)$ is uniquely determined by only one operator $N_{\lambda_{*}}$ with $\lambda_{*} \neq 0$ remains open. This article mainly deals with the case of the Dirichlet-Neumann operator $N_{\mathcal{X}}:=N_{0}$. Section 2 gives an intrinsic interpretation Electrical Impedance Tomography on manifolds, EIT for short, in terms of Inverse-Dirichlet-Neumann problem for twisted Laplacian. In dimension two, this clearly underline how the complex structure of Riemannian surfaces is involved.

Two surfaces in the same conform class which have the same oriented boundary and whose metrics coincides there, need to have the same DirichletNeumann operator. Conversely, Lassas and Uhlmann [21] have proved for a connected $\mathcal{X}$ that the conform class and so the complex structure of $(\mathcal{X}, g)$ is determined by $N_{\mathcal{X}}$. Hence, it is relevant to consider $\mathcal{X}$ as a Riemann surface. In [5], using also the full knowledge of $N_{\mathcal{X}}$, Belishev gives another proof of the above unicity by recovering abstractly $\mathcal{X}$ as the spectre of the algebra of boundary values of functions holomorphic on $\mathcal{X}$ and continuous on $\overline{\mathcal{X}}=\mathcal{X} \cup b \mathcal{X}$. It turns out in our theorems 11 and 2 that only three generic functions on the boundary and their images by $N_{\mathcal{X}}$ are sufficient for unicity to hold and to reconstruct $\mathcal{X}$ by integral Cauchy type formulas. Theorems $3 \mathrm{a}, 3 \mathrm{~b}$ and $3 \mathrm{c}$ deal with characterizations of data of the type $\left(b \mathcal{X}, N_{\mathcal{X}}\right)$ where $\mathcal{X}$ is a Riemann surface.

While the frame of bordered manifolds is sufficient for real analytic boundaries, characterization statements lead to consider a wider class of manifolds. In this article, $(\overline{\mathcal{X}}, \gamma)$ is a Riemann surface with almost smooth boundary if the following holds ${ }^{(1)}: \overline{\mathcal{X}}$ is a compact metrizable topological manifold which is the closure of $\mathcal{X}=\overline{\mathcal{X}} \backslash \gamma, \mathcal{X}$ is a Riemann surface ${ }^{(2)}, h^{2}(\overline{\mathcal{X}})<\infty$, $\gamma$ is a smooth real curve ${ }^{(3)}$ and the set $\overline{\mathcal{X}}_{\text {sing }}$ of points of $\gamma$ where $\overline{\mathcal{X}}$ has not smooth boundary satisfies $h^{1}\left(\overline{\mathcal{X}}_{\text {sing }}\right)=0 ; \overline{\mathcal{X}} \backslash \overline{\mathcal{X}}_{\text {sing }}$ is denoted $\overline{\mathcal{X}}_{\text {reg }}$.

If $(\overline{\mathcal{X}}, \gamma)$ is a Riemann surface with almost smooth boundary, classical results contained in [1] implies a Riemann's existence theorem : a real valued function $u$ of class $C^{1}$ on $\gamma$ has a unique continuous extension $\widetilde{u}$ to $\overline{\mathcal{X}}$ which is harmonic on $\mathcal{X}$, smooth on $\overline{\mathcal{X}}_{\text {reg }}$ and satisfy $\int_{\mathcal{X}} i \partial \widetilde{u} \wedge \bar{\partial} \widetilde{u}<+\infty$. Moreover, $N_{\mathcal{X}} u$ still make sense as the element of the dual space of $C^{1}(\gamma)$ which equals $\partial \widetilde{u} / \partial \nu$ on $\gamma \backslash \overline{\mathcal{X}}_{\text {sing }}$ (see proposition 12).

In the sequel, $\gamma$ is a smooth compact oriented real curve without component reduced to a point, $N$ is an operator from $C^{1}(\gamma)$ to the space of currents on $\gamma$ of degree 0 and order 1 (i.e. functionals on $C^{1} 1$-forms on $\gamma$ ), $\tau$ is a smooth generating section of $T \gamma$ and $\nu$ is another vector field along $\gamma$ such that the bundle $\mathcal{T}$ generated by $\left(\nu_{x}, \tau_{x}\right), x \in \gamma$, has rank $2 ; \gamma$ is assumed to be oriented by $\tau$ and $\mathcal{T}$ by $(\nu, \tau)$.

The inverse Dirichlet-Neumann problem for $(\gamma, N, \mathcal{T})$ is to find, when it exists, an open riemaniann surface $(\mathcal{X}, g)$ with almost smooth boundary $\gamma$ such that for all $x \in \gamma \cap \overline{\mathcal{X}}_{\text {reg }},\left(\nu_{x}, \tau_{x}\right)$ is a positively oriented orthonormal

\footnotetext{
${ }^{1}$ A Stokes formula holds automatically for such manifolds (see lemma 11 in section 3 ).

${ }^{2} h^{d}$ is the $d$-dimensionnial Hausdorff measure.

${ }^{3}$ It could have been possible to allow singularities on $\gamma$ itself but we have avoid it for the sake of simplicity of statements. Likewise, we consider only smooth DN-datas in the sequel.
} 
basis of $T_{x} \overline{\mathcal{X}}$ and for all $u \in C^{1}(\gamma), N u=N_{\mathcal{X}} u$ in the sense of currents. As these conditions do not distinguished between metrics $g$ in a same conformal class, we look after $\mathcal{X}$ as a Riemann surface. The connection between real and complex analysis in the IDN-problem is realized through the operators $L$ and $\theta$ defined for $u \in C^{1}(\gamma)$ by

$$
L u=\frac{1}{2}(N u-i T u) \quad \& \quad \theta u=(L u)\left(\nu^{*}+i \tau^{*}\right)
$$

where $T$ is the tangential derivation by $\tau$ and $\left(\nu_{x}^{*}, \tau_{x}^{*}\right)$ is the dual basis of $\left(\nu_{x}, \tau_{x}\right)$ for every $x \in \gamma$. Note that in the sense of currents, the equality $N u=N_{\mathcal{X}} u$ is equivalent to the identity $\partial \widetilde{u}=\theta u$, the tilde denoting, as through all this article, continuous harmonic extension to $\mathcal{X}$

If $(\overline{\mathcal{X}}, \gamma)$ is a Riemann surface with almost smooth boundary, $g$ is a hermitian metric on $\mathcal{X}$ for which $\left(\tau_{x}, \nu_{x}\right)$ is a positively oriented orthonormal basis of $T_{x}^{*} \mathcal{X}$ for $x \in \gamma$ outside $\sigma=\overline{\mathcal{X}}_{\text {sing }}$ and if $\rho \in C^{0}(\overline{\mathcal{X}}) \cap C^{\infty}(\overline{\mathcal{X}} \backslash \sigma)$ is a defining function of $\gamma$ in $\overline{\mathcal{X}}$, then $\left(\nu^{*}, \tau^{*}\right)=\frac{1}{\left.d \rho\right|_{g}}\left(d \rho, d^{c} \rho\right)$ on $\gamma \backslash \sigma$ where $d^{c}=i(\bar{\partial}-\partial)$ and $\partial \widetilde{u}=(L u)|\partial \rho|_{g}^{-1} \partial \rho=\theta u$ on $\gamma \backslash \sigma$ for all $u \in C^{1}(\gamma)$.

Main hypothesis. In addition to the assumptions on $\gamma$, we consider in all this paper $u_{0}, u_{1}, u_{2} \in C^{\infty}(\gamma)$ three real valued functions only ruled by the main hypothesis that

$$
f=\left(f_{1}, f_{2}\right)=\left(\left(L u_{\ell}\right) /\left(L u_{0}\right)\right)_{\ell=1,2}=\left(\left(\theta u_{\ell}\right) /\left(\theta u_{0}\right)\right)_{\ell=1,2}
$$

is an embedding of $\gamma$ in $\mathbb{C}^{2}$ considered as the complement of $\left\{w_{0}=0\right\}$ in the complex projective plane $\mathbb{C P}_{2}$ with homogeneous coordinates $\left(w_{0}: w_{1}: w_{2}\right)$. Prop. 0 whose proof is omitted shows this is somehow generic :

Proposition 0. Assume $\gamma, u_{0}, u_{1}$ real analytic and that $f_{1}$ is non constant on each connected component of $\gamma$. For any function $u_{2} \in C^{\omega}(\gamma)$, one can construct $v_{2} \in C^{\omega}(\gamma)$, arbitrarily close to $u_{2}$ in $C^{2}$ norm, such that $\left(f_{1},\left(L v_{2}\right) /\left(L u_{0}\right)\right)$ is an embedding of $\gamma$ into $\mathbb{C}^{2}$.

Assuming that $u=\left(u_{\ell}\right)_{0 \leqslant \ell \leqslant 2}$ satisfies the main hypothesis, we set $\theta u=$ $\left(\theta u_{\ell}\right)_{0 \leqslant \ell \leqslant 2}$ and call $(\gamma, u, \theta u)$ a restricted $D N$-datum for an open Riemann surface $\mathcal{X}$ if $\mathcal{X}$ has almost smooth boundary $\gamma,\left.\left(\partial \widetilde{u}_{\ell}\right)\right|_{\gamma \backslash \sigma}=\theta u_{\ell}$ for $0 \leqslant \ell \leqslant 2$ and the well defined meromorphic quotient $F_{\ell}$ of $(1,0)$-forms $\left(\partial \widetilde{u_{\ell}}\right) /\left(\partial \widetilde{u_{0}}\right)$ extends $f_{\ell}$ to $\mathcal{X}$ in the sense that for every $x_{0} \in \gamma, \lim _{x \rightarrow x_{0}, x \in \mathcal{X}} F(x)$ exists and equals $f\left(x_{0}\right)$. If $\gamma$ and $f$ are real analytic, this last property holds automatically.

We define an isomorphism between two Riemann surfaces with almost smooth boundary $(\overline{\mathcal{X}}, \gamma)$ and $\left(\overline{\mathcal{X}^{\prime}}, \gamma^{\prime}\right)$ as a map from $\overline{\mathcal{X}}$ to $\overline{\mathcal{X}^{\prime}}$ which realizes a complex analytic isomorphism between $\mathcal{X}$ and $\mathcal{X}^{\prime}$. As the definition of a Riemann surface with almost smooth boundary implies that its boundary is locally a Jordan curve in its double which is the compact Riemann surface obtained by gluing along its boundary its conjugate (see [1]), a theorem of Caratheodory implies that if $\Phi: \mathcal{X} \rightarrow \mathcal{X}^{\prime}$ is a complex analytic isomorphism, $\Phi$ and $\Phi^{-1}$ extend continuously to $\gamma$ and $\gamma^{\prime}$ so that $\Phi$ becomes a homeomorphism from $\overline{\mathcal{X}}$ to $\overline{\mathcal{X}^{\prime}}$. Hence, $\Phi$ is a diffeomorphism between manifolds with boundary from $\overline{\mathcal{X}}_{\text {reg }} \cap \Phi^{-1}\left(\overline{\mathcal{X}}_{\text {reg }}\right)$ to ${\overline{\mathcal{X}^{\prime}}}_{\text {reg }} \cap \Phi\left(\overline{\mathcal{X}}_{\text {reg }}\right)$. 
The first theorem of this article is a significative improvement of results in [6] 21] on how unique $\mathcal{X}$ can be when a restricted DN-datum is specified.

Theorem 1. Assume that $\mathcal{X}$ and $\mathcal{X}^{\prime}$ are open Riemann surfaces with restricted $D N$-datum $(\gamma, u, \theta u)$. Then, there is an isomorphism of Riemann surfaces with almost smooth boundary between $\mathcal{X} \cup \gamma$ and $\mathcal{X}^{\prime} \cup \gamma$ whose restriction on $\gamma$ is the identity.

Remarks. 1. If $E \subset \gamma$ and $h^{1}(E \cap c)>0$ for each connected component $c$ of $\gamma$, meromorphic functions are uniquely determined by their values on $E$ and it follows that th. 1 conclusions hold when $N_{\mathcal{X}^{\prime}} u_{\ell}=N_{\mathcal{X}} u_{\ell}$ is ensured only on $E$ and the meromorphic functions $\left(\partial \widetilde{u}_{\ell}\right) /\left(\partial \widetilde{u}_{0}\right)$ are continuous near $\gamma$. This includes [21, th. 1.1.i] which is stated for a connected $\mathcal{X}$.

2. The proof of theorem 11 also contains the fact that two connected compact Riemann surfaces $\mathcal{Z}$ and $\mathcal{Z}^{\prime}$ are isomorphic when they share a same real smooth curve $\gamma$ which can be embedded into $\mathbb{C}^{2}$ by a map which extends meromorphically both to $\mathcal{Z}$ and $\mathcal{Z}^{\prime}$ and continuously near $\gamma$.

The assumption on $u_{0}, u_{1}$ and $u_{2}$ is used only to ensure that the map $f$ defined by (1.2) is an embedding of $\gamma$ into $\mathbb{C}^{2}$ extending meromorphically to $\mathcal{X}$ into $F=\left(\partial \widetilde{u}_{\ell} / \partial \widetilde{u}_{0}\right)_{\ell=1,2}$. Moreover, theorem 10 in section 3 implies that if $\mathcal{X}$ has almost smooth boundary and solves the IDN-problem, the map $F$ enable to see $\overline{\mathcal{X}}$ as a normalization of the closure of a complex curve $^{(4)}$ of $\mathbb{C P}_{2} \backslash f(\gamma)$ uniquely determined by $\gamma$. This shows that in each characterization theorem 3a, 3b, 3a, the constructed Riemann surface is, up to isomorphism, the only one which has a chance to solve the IDN-problem.

Our next result explains how to recover $F(\mathcal{X})$ and $\partial \widetilde{u_{\ell}}$ from $\theta u_{\ell}$ and the intersection of $F(\mathcal{X})$ with the lines $\Delta_{\xi}=\left\{z_{2}:=\frac{w_{2}}{w_{0}}=\xi\right\}, \xi \in \mathbb{C}$. Desingularization arguments enable then the reconstruction of $\mathcal{X}$ from $F(\mathcal{X})$.

Theorem 2. If $\mathcal{X}$ is an open Riemann surface with restricted DN-datum $(\gamma, u, \theta u)$, the following holds :

1) The map $f$ defined by (1.耳) has a meromorphic extension $F$ to $\mathcal{X}$ and there are discrete sets $\mathcal{A}$ and $\mathcal{B}$ in $\mathcal{X}$ and $\mathcal{Y}=F(\mathcal{X}) \backslash f(\gamma)$ respectively such that $F: \mathcal{X} \backslash \mathcal{A} \rightarrow \mathcal{Y} \backslash \mathcal{B}$ is one to one.

2) Almost all $\xi_{*} \in \mathbb{C}$ has a neighborhood $W_{\xi_{*}}$ such that for all $\xi$ in $W_{\xi_{*}}$, $\mathcal{Y}_{\xi}=\mathcal{Y} \cap \Delta_{\xi}=\underset{1 \leqslant j \leqslant p}{\cup}\left\{\left(h_{j}(\xi), \xi\right)\right\}$ where $h_{1}, \ldots, h_{p}$ are $p$ mutually distinct holomorphic functions on $W_{\xi_{*}}$ whose symmetric functions $S_{h, m}=\sum_{1 \leqslant j \leqslant p} h_{j}^{m}$ are recovered by the Cauchy type integral formulas

$\left(E_{m, \xi}\right) \quad \frac{1}{2 \pi i} \int_{\gamma} \frac{f_{1}^{m}}{f_{2}-\xi} d f_{2}=S_{h, m}(\xi)+P_{m}(\xi), m \in \mathbb{N}$,

where $P_{m}$ is a polynomial of degree at most $m$. More precisely, the system $E_{\xi}=\left(E_{m, \xi_{\nu}}\right)_{\substack{0 \leqslant m \leqslant B-1 \\ o \leqslant \nu \leqslant A-1}}$ enables explicit computation of $h_{j}\left(\xi_{\nu}\right)$ and $P_{m}$ if $A \geqslant B \geqslant 2 p+1$ and $\xi_{0}, \ldots, \xi_{A}$ are mutually distinct points.

3) For almost all $\xi_{*} \in \mathbb{C}, W_{\xi_{*}}$ can be chosen so that $\mathcal{B} \cap \underset{\xi \in W_{\xi_{*}}}{\cup} \mathcal{Y}_{\xi}=\varnothing$ and $\partial \widetilde{u}_{\ell}, 0 \leqslant \ell \leqslant 2$, can be reconstructed in $F^{-1}\left(\underset{\xi \in W_{\xi_{*}}}{\cup} \mathcal{Y}_{\xi}\right)$ from the well defined

\footnotetext{
${ }^{4}$ That is a pure 1-dimensionnal complex analytic subset of an open subset of $\mathbb{C P}_{2}$.
} 
meromorphic quotient $\left(\partial \widetilde{u}_{\ell}\right) /\left(\partial F_{2}\right)$ thanks to the Cauchy type formulas

$$
\left(T_{m, \xi}\right) \quad \frac{1}{2 \pi i} \int_{\gamma} \frac{f_{1}^{m}}{f_{2}-\xi} \theta u_{\ell}=\sum_{1 \leqslant j \leqslant p} h_{j}(\xi)^{m} \frac{\partial \widetilde{u_{\ell}}}{\partial F_{2}}\left(F^{-1}\left(h_{j}(\xi), \xi\right)\right)+Q_{m}(\xi)
$$

where $m$ is any integer and $Q$ is a polynomial of degree at most $m$.

Remark. The number $\alpha$ of connected components of $\mathcal{X}$ can be computed by the following algorithm : let $\gamma_{1}$ be a component of $\gamma$ and let $\lambda_{1}$ be a function which is zero on $\gamma_{j}$ for $j \neq 0$ and non constant on $\gamma_{1}$; then if $\mathcal{X}_{1}$ is the component of $\mathcal{X}$ whose boundary contains $\gamma_{1}, N \lambda_{1} \neq 0$ on each component $\gamma_{1}, \ldots, \gamma_{k}$ of $\gamma$ which with $\gamma_{1}$ are the components of $b \mathcal{X}_{1}$. Iterating this with components $\gamma$ different from $\gamma_{1}, \ldots, \gamma_{k}$, yields a process with $\alpha$ steps.

The numerical resolution of $\left(E_{\xi}\right)$ and the study of its stability requires an estimate of the number $I_{\Delta_{\xi}}$ of points of intersection, multiplicities taken in account, of $\mathcal{Y}$ with $\Delta_{\xi}$. To achieve this, it is sufficient to estimates the number $I_{\Delta}$ of intersection points of $\mathcal{Y}$ with a $\mathbb{C P}_{2}$-line $\Delta$ generic in the sense that $\Delta$ does not contained the germ of a component of $\mathcal{Y}$ near $\gamma$. Indeed, if $L\left(\right.$ resp. $L_{\xi}$ ) denotes a linear homogeneous form defining $\Delta$ (resp. $\Delta_{\xi}$ ),

$$
I_{\Delta_{\xi}}-I_{\Delta}=\frac{1}{2 \pi i} \int_{\gamma}\left(L_{\xi} / L\right)^{-1} d\left(L_{\xi} / L\right) .
$$

Thus, an a priori upper bound of $I_{\Delta}$ for any particular line $\Delta$ would be very useful. This open problem is related, because of the Ahlfors theorem on covering surface, to the computation of the genus $g_{\mathcal{X}}$ of $\mathcal{X}$ from some DNdatum when $\mathcal{X}$ is connected. Under the condition $\gamma$ is connected, Belishev [5] has shown that $2 g_{\mathcal{X}}$ is the rank of $I d+\left(N_{\mathcal{X}} T^{-1}\right)^{2}$ acting on the space of smooth functions on $\gamma$ admitting a smooth primitive, $T^{-1}$ being a primitive operator. Yet, a formula for $g_{\mathcal{X}}$ involving only the action of $N_{\mathcal{X}}$ on a finite generic set of functions has to be found.

The third aspect of the IDN-problem, characterization of what can and should be a DN-datum, have lead us to allow $\mathcal{X}$ to have only almost smooth boundary. Th. 3a below explicitly characterizes the only right candidate for $\mathcal{X}$ while its part $\mathrm{C}$ gives a test which discriminates which $(\gamma, u, \theta u)$ are DN-data and which are not. To perform it, we need a Green function for $\overline{\mathcal{X}}$ relatively to a domain $\mathcal{D}$ of $\mathcal{Z}$ containing $\overline{\mathcal{X}}$, that is a smooth symmetric function $g$ defined on $\mathcal{D} \times \mathcal{D}$ without its diagonal such that each $g(., z)$ is harmonic on $\mathcal{D} \backslash\{z\}$ and has singularity $\frac{1}{2 \pi} \ln$ dist $(., z)$ at $z$, the distance being computed in any hermitian metric on $\mathcal{Z}$.

Theorem 3a. Assume that the main hypothesis is valid and consider

$$
G: \mathbb{C}^{2} \ni\left(\xi_{0}, \xi_{1}\right) \mapsto \frac{1}{2 \pi i} \int_{\gamma} f_{1} \frac{d\left(\xi_{0}+\xi_{1} f_{1}+f_{2}\right)}{\xi_{0}+\xi_{1} f_{1}+f_{2}} .
$$

A. If an open Riemann surface $\mathcal{X}$ has restricted $D N$-datum $(\gamma, u, \theta u)$, then almost all point $\xi_{*}$ of $\mathbb{C}^{2}$ has a neighborhood where one can find mutually 
distinct holomorphic functions $h_{1}, \ldots, h_{p}$ such that

$$
\begin{gathered}
0=\frac{\partial^{2}}{\partial \xi_{0}^{2}}\left(G-\sum_{1 \leqslant j \leqslant p} h_{j}\right) \\
h_{j} \frac{\partial h_{j}}{\partial \xi_{0}}=\frac{\partial h_{j}}{\partial \xi_{1}}, 1 \leqslant j \leqslant p .
\end{gathered}
$$

B. Conversely, assume $\gamma$ is connected and the conclusion of $A$ is satisfied in a connected neighborhood $W_{\xi_{*}}$ of one point $\left(\xi_{0 *}, \xi_{1 *}\right)$. Then, if $\left(\partial^{2} G / \partial \xi_{0}^{2}\right)_{\mid W_{\xi_{*}}} \neq 0$, there is an open Riemann surface $\mathcal{X}$ with almost smooth boundary ${ }^{(5)} \gamma$ where $f$ extends meromorphically. If $\left(\partial^{2} G / \partial \xi_{0}^{2}\right)_{{ }_{W_{*}}}=0$, the same conclusion holds for a suitable orientation of $\gamma$.

C. Assume that $(\overline{\mathcal{X}}, \gamma)$ is a Riemann surface with almost smooth boundary. Let $\mathcal{Z}$ be the double of $\mathcal{X}, \mathcal{D}$ a smooth domain of $\mathcal{Z}$ containing $\overline{\mathcal{X}}$ and $g$ a Green function for $\overline{\mathcal{X}}$ relatively to $\mathcal{D}$. Then, $(\gamma, u, \theta u)$ is actually a restricted $D N$-datum if and only if for any $z \in \mathcal{D} \backslash \overline{\mathcal{X}}$,

$$
\int_{\gamma} u_{\ell}(\zeta) \partial_{\zeta} g(\zeta, z)+g(\zeta, z) \overline{\theta u_{\ell}(\zeta)}=0,0 \leqslant \ell \leqslant 2 .
$$

Remarks. 1. The connectness of $\gamma$ is essentially used to ensure that any possible solution to the IDN-problem has to be connected. Taking in account the remark following th. 2, one may weaken the connectness assumption on $\gamma$ into the requirement that the given $\mathrm{DN}$-datum ensures that possible solutions are connected. Then, the conclusions of th. 3a.B are still true (see the proof).

2. The proof includes that if $\gamma$ and $f$ are real analytic, $(\overline{\mathcal{X}}, \gamma)$ is a manifold with boundary in the classical sense.

3. Emphasizing on $f_{2}$ instead of $f_{1}$, one can consider

$$
G_{2}: \xi \mapsto \frac{1}{2 \pi i} \int_{\gamma} f_{2} \frac{d\left(\xi_{0}+\xi_{1} f_{1}+f_{2}\right)}{\xi_{0}+\xi_{1} f_{1}+f_{2}} .
$$

If $h_{j}$ is linked to $h_{j, 2}$ by $0=\xi_{0}+\xi_{1} h_{j}+h_{j, 2},\left(h_{1}, \ldots, h_{p}\right)$ satisfy (1.5) and (1.4) if and only $\frac{\partial^{2}}{\partial \xi_{0}^{2}}\left(G_{2}-\sum_{1 \leqslant j \leqslant p} h_{j, 2}\right)=0$ and $h_{j} \frac{\partial h_{j, 2}}{\partial \xi_{0}}=\frac{\partial h_{j, 2}}{\partial \xi_{1}}, 1 \leqslant j \leqslant p$.

4. Select $\mathcal{H}=\left\{h_{1}, \ldots, h_{p}\right\}$ satisfying (1.5) and minimal for (1.4). Then, section 5.2 and proposition 14 shows that there is $\tau^{\prime} \subset \delta=f(\gamma)$ such that $h^{1}\left(\tau^{\prime}\right)=0$ and $\mathcal{X}$ is a normalization of the abstract curve $\mathcal{Y} \cup \tau^{\prime}$ where, when $\mathcal{H}=\varnothing, \mathcal{Y}$ is the polynomial hull of $\delta$ in the affine complex plane

$$
\mathbb{C}_{\xi_{*}}^{2}=\left\{w \in \mathbb{C P}^{2} ; \xi_{*} w=\xi_{0 *} w_{0}+\xi_{1 *} w_{1}+w_{2} \neq 0\right\}
$$

and, otherwise, $\mathcal{Y}$ is the analytic extension in $\mathbb{C P}_{2} \backslash \delta$ of the union of the graphs of the functions $\left(1: h_{j}:-\xi_{0}-\xi_{1} h_{j}\right), 1 \leqslant j \leqslant p$. Hence, when $\mathcal{H}$ is minimal, decomposition (1.4) of $G$ is unique up to order and Card $\mathcal{H}$ is the minimal number $p$ for which such a decomposition exists. Moreover, theorem 10 implies that the only Riemann surfaces $\mathcal{X}$ which has a chance to solve the IDN-problem are normalizations of $\mathcal{Y}$.

\footnotetext{
${ }^{5}$ With [18, example 10.5], one can construct smooth restricted DN-datas for which the solution of the IDN-problem is a manifold with only almost smooth boundary.
} 
The vanishing of $\partial^{2} G / \partial \xi_{0}^{2}$ in a connected neighborhood $W_{\xi_{*}}$ of $\xi_{*} \in \mathbb{C}^{2}$ is known to be equivalent to the fact that $\delta=f(\gamma)$ satisfy the classical Wermer-Harvey-Lawson moment condition in ${ }^{(6)} \mathbb{C}_{\xi_{*}}^{2}$ : for all $k_{1}, k_{2} \in \mathbb{N}$, $\int_{\delta} z_{1}^{k_{1}} z_{2}^{k_{2}} d z_{2}=0$ where $z=\left(w_{j} / \xi_{*} w\right)_{j=1,2}$ (see [12, cor. 1.6.2]). It is proved in [29] for the real analytic case and in [0] [18] for the smooth case that for a suitable orientation of $\gamma$, this moment condition guarantees the existence in $\mathbb{C}_{\xi_{*}}^{2} \backslash \delta$ of a unique complex curve $\mathcal{Y}$ with finite mass and boundary $\pm \delta$ in the sense of currents. In [2], Alexander and Wermer have improved this WermerBishop-Harvey-Lawson statement by showing that a closed oriented smooth connected real curve $\delta$ of $\mathbb{C}^{2}$ is, with its given orientation, the boundary, in the sense of currents, of a complex curve of finite mass in $\mathbb{C}_{\xi_{*}}^{2} \backslash \delta$ if and only if $\frac{1}{2 \pi i} \int_{\delta} \frac{d A}{A} \geqslant 0$ for any polynomial $A$ which does not vanish on $\gamma$. Hence, in case $\left(\partial^{2} G / \partial \xi_{0}^{2}\right)_{\mid W_{\xi_{*}}}=0$ it is sufficient to find one polynomial $A$ such that $\int_{f(\gamma)} \frac{d A}{A} \neq 0$ to determine the correct orientation of $\gamma$.

Note that the case $\left(\partial^{2} G / \partial \xi_{0}^{2}\right)_{\mid W_{\xi_{*}}}=0$ occurs only for very special DNdata since it implies that for $\ell=1,2, f_{\ell}$ admits a $\mathbb{C}_{\xi_{*}}^{2}$-valued holomorphic extension to $\mathcal{X}$. Proposition 20 proposes another result of this kind for some other special DN-data when they are available.

To palliate the difficulty of computing Green functions, the theorem below proposes another way to achieve the same goals : select the right candidates for $\mathcal{X}$ and extension of $\theta u_{\ell}$; check this yields a solution.

Theorem 3b. Assume that the main hypothesis is valid. Let $G$ be the function defined by (1.9) and let be $\widetilde{G}$ the form which in $\mathbb{C P}_{2}$ with homogenous coordinates $\eta=\left(\eta_{0}: \eta_{1}: \eta_{2}\right)$ is given by

$$
\widetilde{G}=\sum_{0 \leqslant \ell \leqslant 2} \frac{1}{2 \pi i}\left(\int_{\gamma} \frac{\theta u_{\ell}}{\eta_{0}+\eta_{1} f_{1}+\eta_{2} f_{2}}\right) d \eta_{\ell}=\sum_{0 \leqslant \ell \leqslant 2} \widetilde{G}_{\ell} d \eta_{\ell}
$$

$\boldsymbol{A}$. If an open Riemann surface $\mathcal{X}$ has restricted $D N$-datum $(\gamma, u, \theta u)$, then,

a1) Almost all points $\eta_{*}=\left(\xi_{* 0}: \xi_{* 1}: 1\right)$ of $\mathbb{C P}_{2}$ has a neighborhood where $\widetilde{G}$ can be written as the sum of $p$ holomorphic closed forms $g_{j}=\sum_{0 \leqslant \ell \leqslant 2} g_{j, \ell} d \eta_{\ell}$ such that $\left(h_{j}\right)=\left(g_{j, 1} / g_{j, 0}\right)_{1 \leqslant j \leqslant p}$ satisfy (1.5).

a2) The form $\Theta_{\ell}=\partial \tilde{u}_{\ell}, 0 \leqslant \ell \leqslant 2$, satisfies

$$
\int_{c} \operatorname{Re} \Theta_{\ell}=0
$$

for all $c$ in the first homology group $H_{1}(\mathcal{X})$ of $\mathcal{X}$.

B. b1) Assume $\gamma$ is connected and there is $\eta_{*}=\left(\xi_{* 0}: \xi_{* 1}: 1\right)$ and a connected neighborhood $W_{\xi_{*}}$ of $\xi_{*}=\left(\xi_{* 0}, \xi_{* 1}\right)$ such that (a1) is true for all $\eta \in W_{\eta_{*}}=\left\{\left(\xi_{0}: \xi_{1}: 1\right) ;\left(\xi_{0}, \xi_{1}\right) \in W_{\xi_{*}}\right\}$. Then, there exists an open

\footnotetext{
${ }^{6}$ When $\xi_{*}$ belongs to the connected component of infinity of $\left\{\xi \in \mathbb{C}^{2} ; \forall w \in \delta, \xi w \neq 0\right\}$, this moment condition is equivalent to the moment condition in $\mathbb{C}_{(1,0)}^{2}$ and also to the vanishing of $G$ on this component.
} 
Riemann surface $\mathcal{X}$, topologically bordered by $\gamma$, where $f$ extends meromorphically and each $\theta u_{\ell}$ extends weakly(7) into a meromorphic (1,0)-form $\Theta_{\ell}$ outside a set $\Sigma$ of zero length ${ }^{(8)}$.

b2) In addition to (a1), assume that $\Theta_{\ell}$ satisfies $\int i \Theta_{\ell} \wedge \overline{\Theta_{\ell}}<+\infty$ and 1.8). Then if $\left(\partial^{2} G / \partial \xi_{0}^{2}\right)_{W_{\xi_{*}}} \neq 0,(\overline{\mathcal{X}}, \gamma)$ is a manifold with almost smooth boundary ; the same conclusion holds when $\left.\widetilde{G}\right|_{W_{\eta_{*}}}=0$ if $\gamma$ has a suitable orientation. If $\left(\partial^{2} G / \partial \xi_{0}^{2}\right)_{W_{\xi_{*}}}=0$ but $\left.\widetilde{G}\right|_{W_{\eta_{*}}} \neq 0$, then either $\mathcal{X}$ is a domain with boundary $\gamma$ in a normalization of an algebraic curve of $\mathbb{C P}_{2}$, either $\overline{\mathcal{X}}$ is a compact Riemann surface where $\gamma$ is a slit ${ }^{(9)}$. In all cases, $u_{\ell}$ admits a continuous extension $\widetilde{u_{\ell}}$ to $\overline{\mathcal{X}}$ which is harmonic in $\mathcal{X}$ and such that $\Theta_{\ell}=\partial \widetilde{u_{\ell}}$, which means that $N u_{\ell}$ is actually the DN-datum of $\mathcal{X}$ for $u_{\ell}$.

Remarks. 1. When (a1) holds, $h_{j, 2}=\frac{g_{j, 2}}{g_{j, 0}}$ verify $h_{j} \frac{\partial h_{j, 2}}{\partial \xi_{0}}=\frac{\partial h_{j, 2}}{\partial \xi_{1}}, 1 \leqslant j \leqslant p$.

2. Formulas $\left(E_{m . \xi}\right)$ and $\left(T_{m . \xi}\right)$ enable direct reconstruction of a projective presentation of $\mathcal{X}$ and forms $\Theta_{\ell}$.

Theorem $3 \mathrm{~b}$ is obtained by a normalization of a singular version of the IDN-problem which is more explicit. When $\mathcal{X}$ is smooth, the harmonicity of a distribution $U$ is equivalent to the fact that $\partial U$ is holomorphic. For the case where $\mathcal{X}$ is a complex curve of an open set in $\mathbb{C P}_{2}$, we need two of the several non equivalent definitions of holomorphic (1,0)-forms.

At first, we use the weakly holomorphic forms introduced by Rosenlicht 25] which can be defined as meromorphic (1,0)-forms $\psi$ such that $\psi \wedge[\mathcal{X}]$ is $\bar{\partial}$-closed current of $\mathbb{C P}_{2}$. Such forms $\psi$ are also characterized by the fact that $p_{*} \psi$ is a usual holomorphic $(1,0)$-form for any holomorphic proper function $p: \mathcal{X} \rightarrow \mathbb{C}$. A distribution $U$ is defined as weakly harmonic if $\partial U$ is weakly holomorphic.

Assume now $\mathcal{X}$ lies in $\mathbb{C P}_{2}$ and that $\mathcal{X}$ is bounded in the sense of currents by $\gamma$. A distribution $U$ on $\mathcal{X}$ is said almost smooth up to the boundary if it is the case near each $p \in \gamma$ where $(\overline{\mathcal{X}}, \gamma)$ is a manifold with boundary and if $U$ has a restriction on $\gamma$ in the sense of currents. When $u$ is a smooth function on $\gamma$, a weakly harmonic extension of $u$ to $\mathcal{X}$ is a weakly harmonic distribution $U$ almost smooth up to boundary whose restriction on $\gamma$ is $u$. Since two weakly harmonic extension $U_{1}$ and $U_{2}$ of $u$ to $\mathcal{X}$ are equal when $\partial U_{1}=\partial U_{2}$ on $\gamma$ in the sense of currents, we consider a weak CauchyDirichlet problem : a data is a smooth function $u$ on $\gamma$ and a smooth section $\lambda$ of $T_{\gamma}^{*} \mathcal{X}$; a solution is a weakly harmonic function $U$ almost smooth up to $\gamma$ such that $u=\left.U\right|_{\gamma}$ and $\lambda=(\partial U)_{\gamma}$ in the sense of currents ; when it exists, such an $U$ is unique and is denoted $\widetilde{u}$ as any harmonic extension in this article. In connection with this notion, we define a weak restricted

\footnotetext{
${ }^{7}$ It means that $\int_{\gamma} \varphi \theta u_{\ell}=\int_{\mathcal{X}} d\left(\varphi \Theta_{\ell}\right)=\int_{\mathcal{X}}(\bar{\partial} \varphi) \wedge \Theta_{\ell}$ holds for any Lipschitz function $\varphi$ on $\overline{\mathcal{X}}$ which is a holomorphic function of $f$ near points of $\Sigma$ and singular points of $(\overline{\mathcal{X}}, \gamma)$; if $(\overline{\mathcal{X}}, \gamma)$ is a manifold with boundary, this definition means that $\left.\Theta_{\ell}\right|_{\gamma}=\theta u_{\ell}$ in the usual sense.

${ }^{8}$ Basing on [11, example 1], one can construct examples where (a1) is satisfied while the weak extension $\Theta_{\ell}$ has essential singularities on some zero length set $\Sigma$.

${ }^{9}$ This means that $\overline{\mathcal{X}} \backslash \gamma$ is connected.
} 
data as a triplet $(\gamma, u, \theta u)$ where $u=\left(u_{\ell}\right)_{0 \leqslant \ell \leqslant 2}\left(\right.$ resp. $\left.\theta u=\left(\theta u_{\ell}\right)_{0 \leqslant \ell \leqslant 2}\right)$ is a triplet of smooth functions (resp. (1,0)-forms) on $\gamma$ such that $\theta u_{\ell}=\left(\partial \widetilde{u}_{\ell}\right)_{\gamma}$ in the sense of currents.

The weak CD-problem has its own interest and arise naturally in the proof of th. 3b. However, the original IDN-problem requires a more restrictive notion of harmonicity. According to Griffiths [15], holomorphic forms (resp. harmonic functions) are by definition push forward of holomorphic forms (resp. harmonic functions) on a normalization of $\mathcal{X}$. Equivalently, a real function $U$ on $\mathcal{X}$ is harmonic if and only if $U$ is harmonic in the regular part $\mathcal{X}_{\text {reg }}$ of $\mathcal{X}$ and $\int_{\mathcal{X}_{\text {reg }}} i \partial U \wedge \bar{\partial} U<+\infty$. This notion is close in spirit to a Riemann characterization of the harmonic function with given boundary value $u$ as the smooth function extending $u$ to $\overline{\mathcal{X}}$ and minimizing the preceding integral. We can now state a singular version of th. $3 \mathrm{~b}$.

Theorem 3c. Consider in $\mathbb{C P}_{2} \backslash\left\{w_{0}=0\right\}$ a smooth oriented real curve $\gamma$, three functions $u_{0}, u_{1}, u_{2}$ in $C^{\infty}(\gamma)$ and $\theta_{0}, \theta_{1}, \theta_{2}$ three smooth sections of $\Lambda^{1,0} T_{\gamma}^{*} \mathbb{C P}_{2}$ such that $d u_{\ell}=2 \operatorname{Re} \theta_{\ell}, 0 \leqslant \ell \leqslant 2$ and linked by the relations $\theta_{1}=z_{1} \theta_{0}, \theta_{2}=z_{2} \theta_{0}$. Let $G$ and $\widetilde{G}$ be the form given by (1.3) and (1.7) but with $\left(f_{1}, f_{2}\right)=\left(z_{1}, z_{2}\right)$.

A. Assume $\gamma$ bounds, in the sense of currents, a complex curve $\mathcal{X}$ of $\mathbb{C P}_{2} \backslash \gamma$ which has finite volume and weak restricted DN-datum $(\gamma, u, \theta u)$. Then,

a1) The conclusions of theorem 30.A.a1 are valid.

a2) The form $\Theta_{\ell}=\partial \widetilde{u_{\ell}}$ satisfies (1.8) for all $c$ in $H_{1}\left(\mathcal{X}_{\mathrm{reg}}\right)$.

B. b1) Conversely, assume that $\gamma$ is connected and that (a1) is valid for one point $\eta_{*}=\left(\xi_{0 *}: \xi_{1 *}: 1\right)$. Then, there is in $\mathbb{C P}_{2} \backslash \gamma$ a complex curve $\mathcal{X}$ of finite mass where each $\theta_{\ell}$ extends weakly on $\mathcal{X}$ into a weakly holomorphic $(1,0)$-form $\Theta_{\ell}$. Moreover, if $\left(\partial^{2} G / \partial \xi_{0}^{2}\right)_{\mid W_{\xi_{*}}} \neq 0$, then $\mathcal{X}$ has boundary $\gamma$ in the sense of currents ; the same conclusion holds if $\left.\widetilde{G}\right|_{W_{\eta_{*}}}=0$ but for a suitable orientation of $\gamma$. If $\left(\partial^{2} G / \partial \xi_{0}^{2}\right)_{\left.\right|_{\xi_{*}}}=0$ but $\left.\widetilde{G}\right|_{W_{\eta_{*}}} \neq 0$, either $\mathcal{X}$ is a domain in an algebraic curve of $\mathbb{C P}_{2}$ and has boundary $\gamma$ in the sense of currents, either $\overline{\mathcal{X}}$ itself is an algebraic curve of $\mathbb{C P}_{2}$ where $\gamma$ is a slit.

b2) If in addition (1.8) is satisfied by $\Theta_{\ell}$ for all $c \in H_{1}\left(\mathcal{X}_{\text {reg }}\right)$, then $u_{\ell}$ has $a$ (unique) weakly harmonic extension $\widetilde{u_{\ell}}$ and $\Theta_{\ell}=\partial \widetilde{u_{\ell}}$. If $\Theta_{\ell}$ also satisfy $\int_{\mathcal{X}_{\text {reg }}} i \Theta_{\ell} \wedge \overline{\Theta_{\ell}}<+\infty$, then $\widetilde{u}_{\ell}$ is harmonic.

Remark. It is possible that $\mathcal{X}$ has zero boundary in the sense of currents. This occurs only in the exceptional case where $\overline{\mathcal{X}}$ is a compact complex curve of $\mathbb{C P}_{2}$ and (so is algebraic) where $\gamma$ is a slit. In the other cases, $\mathcal{X}$ has boundary $\pm \gamma$ in the sense of currents and a result of Chirka 10] gives that outside a zero one Hausdorff dimensional subset, $(\overline{\mathcal{X}}, \pm \gamma)$ is locally a manifold with boundary.

The proofs of the preceding theorems are given in sections three to five. They use the results on the complex Plateau problem started in [29] [7], developed in [18][17] [11] for $\mathbb{C}^{n}$ and in [20] 12] 19] for $\mathbb{C P}_{n}$.

The non constructive existence criterions of theorems 3a, $3 \mathrm{~b}$ and $3 \mathrm{a}$ may incite to seek a less general but more effective characterization. It has been 
already mentioned after theorem 3 a that in the special case $p=0$, the condition $\left(\partial^{2} G / \partial \xi_{0}^{2}\right)_{\mid W_{\xi_{*}}}=0$ together with the Alexander-Wermer moment criterion gives an effective tool but only when special DN-data are at hand.

For $p>0$, the main result of [12] is that conditions of type (1.4) and (1.5) characterize the fact that a given closed smooth and orientable real chain $\gamma$ of $\mathbb{C P}_{2}$ is, with adequate orientation, the boundary of some holomorphic chain of $\mathbb{C P}_{2} \backslash \gamma$. These conditions have been qualified as mysterious in [19] because the functions satisfying these relations are produced "deus ex machina". The following criterion, which completes for a closed connected curve $\gamma$ the one of [12], is obtained in [19]: Suppose that the second coordinate $f_{2}$ of $\mathbb{C}^{2}$ does not vanish on $\gamma$, then there exists in $\mathbb{C P}_{2} \backslash \gamma$ a connected complex curve $\mathcal{X}$ with boundary $\pm \gamma$ in the sense of currents if and only if there exist $p \in \mathbb{N}$ and $A_{d}$ in the space $\mathcal{O}(d)$ of holomorphic homogeneous polynomials of order $d, 1 \leqslant d \leqslant p$, such that for $\xi_{0}$ in some neighborhood of $0, C_{m}\left(\xi_{0}\right)=\frac{1}{2 \pi i} \int_{\gamma} \frac{f_{1}^{m}}{f_{2}+\xi_{0}} d f_{2}$ satisfies

$$
\begin{aligned}
C_{k} & =Q_{k, p}\left(C_{1}, \ldots, C_{p}\right) \bmod \mathcal{O}(k), k>p \\
C_{d}\left(\xi_{0}\right) & =\sum_{k>d} \frac{\left(-\xi_{0}\right)^{k}}{2 \pi i} \int_{\gamma} \frac{f_{1}^{d}}{f_{2}^{k+1}} d f_{2}+A_{d}\left(\xi_{0}\right), 1 \leqslant d \leqslant p
\end{aligned}
$$

where $Q_{k, p}$ are universal homogeneous polynomials.

In section 6.1, theorem 3 a is develop for $p>0$ into theorem 1 below which gives a more effective criterion for the Plateau problem in $\mathbb{C P}_{2}$ and also for the IDN-problem. This new criterion follows from considerations on sums of shock wave functions modulo affine functions in $\xi_{0}$. Even if decompositions in sum of shock wave functions are studied for $\xi_{0}$-affine functions, theorem 4 does not consider the case where $G$ is of that type since it corresponds to a plain case of (1.4).

Notations for theorem 4 : if $H$ and $u$ are holomorphic functions on a simply connected domain $D$, we set $\mathcal{D}_{H}=\frac{\partial}{\partial \xi_{1}}-\frac{\partial H}{\partial \xi_{0}}$ and denote by $\mathcal{L}_{H} u$ the unique function $v \in \mathcal{O}(D)$ such that $\partial v / \partial \xi_{0}=\mathcal{D}_{H} u$ and $v(0,)=.0 ; \pi_{1}$ is the projection $\left(\xi_{0}, \xi_{1}\right) \mapsto \xi_{1}$.

Theorem 4. Let $f$ be defined by (1.9) and consider the function $G$ defined by (1.3). We assume that $\gamma$ is connected and that $f_{2}$ does not vanish on $\gamma$ so that $G$, which is assumed to be not affine in $\xi_{0}$, is defined in a simply connected neighborhood $D$ of 0 in $\mathbb{C}^{2}$.

$\boldsymbol{A} /$ If $(\overline{\mathcal{X}}, \gamma)$ is a Riemann surface with almost smooth boundary where $f$ extends meromorphically, then the following assertions hold for $G$

(1) There is $p \in \mathbb{N}^{*}$ and holomorphic functions $a, b, \lambda_{1}, \ldots, \lambda_{p-1}$ on $\Delta=$ $\pi_{1}(D)$ such that the integro-differential equation

$$
-\mathcal{D}_{G+L} \mathcal{L}_{G+L}^{p-1}(G+L)+\sum_{1 \leqslant j \leqslant p-1} \mathcal{D}_{G+L} \mathcal{L}_{G+L}^{p-1-j} \tilde{\lambda}_{j}=0,
$$

is valid with $L=\xi_{0} \otimes a+1 \otimes b$ and $\widetilde{\lambda}_{j}=1 \otimes \lambda_{j}, 1 \leqslant j \leqslant p$.

(2) For $s_{k}=-\mathcal{L}_{G+L}^{k-1} G+\mathcal{L}_{G+L}^{k-2} \widetilde{\lambda}_{1}+\cdots+\mathcal{L}_{G+L}^{0} \widetilde{\lambda}_{k-1}, 1 \leqslant k \leqslant p$, the discriminant of $T_{\xi_{0}, \xi_{1}}=X^{p}+\sum_{1 \leqslant k \leqslant p} s_{k}\left(\xi_{0}, \xi_{1}\right) X^{p-k}$ does not vanish identically in $D$. 
(3) $G=-s_{1}-L$

(4) There is $q \in \mathbb{N}, \alpha, \beta \in \mathbb{C}_{q}\left[\xi_{1}\right]$ such that $\alpha(0)=0$, $\operatorname{deg} \beta<q$ and $a=\frac{\alpha^{\prime}}{1-\alpha}, b=\frac{\beta}{1-\alpha}$

Moreover, if $p$ is the least integer such that (1-2-3) assertions holds, $(\gamma, f)$ uniquely determines $\left(a, b, \lambda_{1}, \ldots, \lambda_{p-1}\right)$.

B/ Assume (1-2-3) holds for some $p \in \mathbb{N}^{*}$. Then, there exists an open Riemann surface $\mathcal{X}$ such that $\overline{\mathcal{X}}=\mathcal{X} \cup \gamma$ is a manifold with almost smooth boundary where $f$ extends meromorphically. Moreover, (4) holds.

Remarks. 1. Non unicity of $\left(a, b, \lambda_{1}, \ldots, \lambda_{p-1}\right)$ solving (1-2-3) means that $\mathcal{X}$ exists but $p$ is not minimal.

2. It is possible that regardless its orientation, $\gamma$ is the almost smooth boundary of an open Riemann surface $\mathcal{X}$ where $f$ extends meromorphically. It is the case when $\gamma$ cuts a compact Riemann surface $\mathcal{Z}$ into two smooth domains and $f$ is the restriction to $\gamma$ of an analytic map from $\mathcal{Z}$ to $\mathbb{C P}_{2}$.

\section{InTRINSiC EIT on RIEMANN SURFACES}

The Inverse Dirichlet-Neuman problem, which goes back to Calderon 9] and which is called now Electrical-Impedance-Tomography problem, can be sketch like this : suppose that a bounded domain $\mathcal{X}$ in $\mathbb{R}^{2}$ or $\mathbb{R}^{3}$ is an ohmic conductor which means that the density of current $j$ it may have is proportional (in isotropic cases) to the electrical field $e=\nabla U$ where $U$ is an electrical potential. The scalar function $\sigma$ such that $j=\sigma e$ is then called the conductivity of $\mathcal{X} ; \rho=1 / \sigma$ is the resistivity. When there is no time dependence and no source or sink of current, the equation $\operatorname{div} j=0$ holds and Calderon's problem is then to recover $\sigma$ on the whole of $\mathcal{X}$ from the operator $C^{\infty}(\gamma) \ni u \mapsto(\sigma \nabla \widetilde{u})_{\gamma}, \widetilde{u}$ being the unique solution of $\operatorname{div}(\sigma \nabla \widetilde{u})=0$ with boundary value $u$.

In what follows, linking the Calderon problem to the Belishev problem mentioned in the introduction, we formulate the EIT-problem for a more general setting than the case of domains in $\mathbb{R}^{n}$. The second part of this section, despite the fact it is also quite elementary, seems to be new and underline how complex structure is involved in the dimension two case.

General dimension. Assume that $\mathcal{X}$, an open oriented bordered manifold of dimension $n$ with boundary $\gamma$, is given with a volume form $\mu$ and a conductivity $\sigma$ modelled as a tensor from $T^{*} \mathcal{X}$ to $\Lambda^{n-1} T^{*} \mathcal{X}$ (see [27]). The gradient associated to $\sigma$ relatively to $\mu$ is the differential operator which to any $f \in C^{1}(\mathcal{X})$ associates the tangent vector field $\nabla_{\mu, \sigma} f$ characterized by

$$
\left.\left(\nabla_{\mu, \sigma} f\right)\right\lrcorner \mu=\sigma(d f)
$$

where $\lrcorner$ is the interior product. When $U \in C^{1}(\mathcal{X})$ is some given potential, the density of physical current $J$ is by definition ${ }^{(10)}$

$$
J=\nabla_{\mu, \sigma} U .
$$

If $\mathcal{X}$ has no source or sink of currents and if $U$ has no time dependence, the flux of current through the boundary of any domain is zero. Using Stokes

\footnotetext{
${ }^{10}$ That $\nabla_{\mu, \sigma} U$ truly models the density of current is the assumption of Ohm's law.
} 
formula, this can be modeled by the simplified Maxwell equation

$$
0=\operatorname{div}_{\mu} J=\operatorname{div}_{\mu}\left(\nabla_{\mu, \sigma} U\right)
$$

where $\operatorname{div}_{\mu}$ is ${ }^{(11)}$ the divergence with respect to the volume form $\mu$. Going back to the definition of gradient and divergence, we see that (2.1) is equivalent to the intrinsic equation formulated in [27] for domains in $\mathbb{R}^{n}$

$$
d \sigma(d U)=0
$$

Since $\mu$ is no longer involved, the usual DN-operator has to be replaced by the operator $\Theta$ which to $u \in C^{1}(\gamma)$ associates $\sigma(d u)_{\gamma}$ which is a section of $\Lambda^{n-1} T_{\gamma}^{*} \mathcal{X}$. The Electrical Impedance Tomography problem, is then to reconstruct $(\mathcal{X}, \sigma)$ from its DN-map $\Theta$. Of course, the two other aspects of this problem, unicity and characterization, also has to be studied.

The problem in such a generality is still widely open ; almost all publications are about domains in $\mathbb{R}^{3}$. In such a case, (2.1) is generally written in euclidean global coordinates. However, when $\mathcal{X}$ is a manifold, (2.2) yields the same equation in any chart $(W, x)$; setting $\sigma d x_{j}=\sum_{1 \leqslant k \leqslant n} \sigma_{k j}(-1)^{k} d x_{\widehat{k}}$ with $d x_{\widehat{k}}=\underset{j \neq k}{\wedge} d x_{j}$, (2.2) becomes

$$
\sum_{1 \leqslant k \leqslant n} \sum_{1 \leqslant j \leqslant n} \frac{\partial}{\partial x_{k}}\left(\sigma_{k, j} \frac{\partial U}{\partial x_{j}}\right)=0
$$

When the conductivity $\sigma$ is symmetric $(\sigma(a) \wedge b \equiv \sigma(b) \wedge a)$ and invertible tensor, it is possible to design a natural metric $g_{\mu, \rho}$ associated to the resistivity map $\rho=\sigma^{-1}$ by the well defined quotient of $n$-forms :

$$
g_{\mu, \sigma^{-1}}(t)=\frac{\left.\left.\sigma^{-1}(t\lrcorner \mu\right) \wedge(t\lrcorner \mu\right)}{\mu}, t \in T \mathcal{X} .
$$

If $(W, x)$ is any coordinates chart for $\mathcal{X}$, a direct calculus in $x$-coordinates shows that for $t=\Sigma t_{k} \partial / \partial x_{k}$ (2.4) becomes

$$
g_{\mu, \rho}(t)=\sum_{k, \ell} t_{\ell} t_{k} \lambda \rho_{k, \ell}
$$

where $\left(\rho_{k, \ell}\right)$ is the matrix of the resistivity $\rho=\sigma^{-1}$ when at any given point $z$ the chosen basis for $\Lambda^{n-1} T_{z}^{*} \mathcal{X}$ and $T_{z}^{*} \mathcal{X}$ are $\left((-1)^{k} d x_{\widehat{k}}\right)$ and $\left(d x_{k}\right)$ respectively. When $\left(\sigma_{j, k}\right)$ is positive definite, $g_{\mu, \rho}$ is a metric on $\mathcal{X}$.

When $n \geqslant 3$, there is a specially adequate choice of metric and volume.

Proposition 5. Assume $n \geqslant 3$. Then one can correctly design a global volume form $\mu$ by letting it be defined by $\mu=\left[\operatorname{det}\left(\rho_{k, \ell}\right)\right]^{\frac{-1}{n-2}} d x_{1} \wedge \cdots \wedge d x_{n}$ in any coordinates chart $(W, x)$ for $\mathcal{X}$. For this specific volume form, $\sigma$ is the Hodge star operator of $g_{\mu, \rho}$ and $\mu$ is the riemannian volume form of $g_{\mu, \rho}$.

This statement, already pointed out by Bossavit and Lee-Uhlmann (see [3] and [21]) for domains in affine spaces, follows from calculus in coordinates.

The interest of proposition 5 is to state the strict equivalence between the IDN-problem for riemannian manifolds and the EIT-problem when $n \geqslant$ 3. When $\operatorname{dim} \mathcal{X} \geqslant 3$ and $\overline{\mathcal{X}}$ is a riemannian real analytic manifold with

\footnotetext{
${ }^{11}$ If $t$ is a differentiable vector field, $\operatorname{div}_{\mu} t$ is defined by $\left.d(t\lrcorner \mu\right)=\left(\operatorname{div}_{\mu} t\right) \mu$.
} 
boundary, Lassas and Uhlmann have proved in 21] that the DN-operator uniquely determine $\mathcal{X}$ and its metric.

The two dimensional case. We now assume $n=2$ and $\sigma=\rho^{-1}$ is symmetric and positive so that $\left(\mathcal{X}, g_{\mu, \rho}\right)$ becomes a riemannian manifold whose volume form is thereafter denoted by $V_{\mu, \rho}$. Let us emphasize the complex structure associated to the conformal class of $\left(\mathcal{X}, g_{\mu, \rho}\right)$ by choosing isothermal coordinates charts, that is holomorphic charts (see e.g. [28]). In such a chart $(W, z)$,

$$
g_{\mu, \rho}=\kappa_{\mu, \rho}(d x \otimes d x+d y \otimes d y)=\operatorname{Re}\left(\kappa_{\mu, \rho} d z \otimes d \bar{z}\right)
$$

where $x=\operatorname{Re} z, y=\operatorname{Im} z$ and $\kappa_{\mu, \rho} \in C^{1}\left(W, \mathbb{R}_{+}^{*}\right)$. Hence, in these coordinates, $\left(\sigma_{k, \ell}\right)=s \operatorname{diag}(1,1)$ with $\kappa_{\mu, \rho}=\lambda / s$ and $\lambda \in C^{1}\left(W, \mathbb{R}_{+}^{*}\right)$ is defined by $\mu=\lambda d x \wedge d y=\lambda \frac{i}{2} d z \wedge d \bar{z}$. Note that $s$ is a global positive function on $\mathcal{X}$ since it is the well defined quotient of volume forms :

$$
s=\mu / V_{\mu, \rho}
$$

Note also that $s$ does not depend on $\mu$ and that (2.2) finally evolutes into

$$
d\left(s d^{c} U\right)=0
$$

where $d^{c}=i(\bar{\partial}-\partial), \bar{\partial}$ and $\partial$ being the usual global differential operators associated to the complex structure of the conformal class of $\left(\mathcal{X}, g_{\mu, \rho}\right)$. Hence, we have proved the following which generalizes a result written by Sylvester [27 for domains in $\mathbb{R}^{2}$.

Proposition 6. Let $\mathcal{X}$ be a real two dimensional manifold equipped with a symmetric and positive tensor $\sigma: T^{*} \mathcal{X} \rightarrow T^{*} \mathcal{X}$. Then, there is a complex structure on $\mathcal{X}$ and $s \in C^{1}\left(\mathcal{X}, \mathbb{R}_{+}^{*}\right)$, called scalar conductivity, such that 2.8) is equivalent to 2.8.

The beginning of this paper has shown that the data $\partial U / \partial \nu$ is equivalent to the data $(\partial U)_{\gamma}$ which don't involve any metric. Since the knowledge of $(\partial U)_{\gamma}$ is equivalent to the knowledge of $\left(s d^{c} U\right)_{\gamma}$, we consider $\left(s d^{c} U\right)_{\gamma}$ as the DN-datum. We can now state an intrinsic IDN-problem for two dimensional ohmic conductors ; for the sake of simplicity, we limit ourselves to manifolds with boundary and smooth datas.

A two dimensional ohmic conductor is a couple $(\mathcal{X}, \rho)$ where $\mathcal{X}$ is an open oriented bordered two dimensional real surface (with boundary $\gamma$ ), the conductivity $\sigma=\rho^{-1}$ is a positive definite tensor from $T^{*} \mathcal{X}$ to $T^{*} \mathcal{X}$ and $\mathcal{X}$ is equipped with the complex structure associated to the riemannian metric $g_{\mu, \rho}$ defined by (2.4) where $\mu$ is any volume form of $\mathcal{X}$. In this setting, the scalar conductivity is the function $s=\mu / V_{\mu, \rho}$ where $V_{\mu, \rho}$ is the volume associated to $g_{\mu, \rho}$. The DN-operator is the operator $\theta_{\mathcal{X}, \rho}$ defined by

$$
\theta_{\mathcal{X}, \rho}: C^{1}(\gamma) \ni u \mapsto\left(s d^{c} \widetilde{u}\right)_{\gamma} \in T_{\gamma}^{*} \mathcal{X}
$$

where $\widetilde{u}$ is the unique solution of the following Dirichlet problem

$$
\left.U\right|_{\gamma}=u \quad \& \quad d\left(s d^{c} U\right)=0 .
$$

The IDN-problem associated to this setting is threefold :

Unicity. Assume that two dimensional ohmic conductors $(\mathcal{X}, \rho)$ and $\left(\mathcal{X}^{\prime}, \rho^{\prime}\right)$ share the same boundary $\gamma$ and the same DN-operator $\theta$. Is it true that 
there is a diffeomorphism $\varphi: \overline{\mathcal{X}} \rightarrow \overline{\mathcal{X}^{\prime}}$ between manifolds with boundaries such that $\varphi: \mathcal{X} \rightarrow \mathcal{X}^{\prime}$ is analytic and $s=s^{\prime} \circ \varphi$ where $s$ and $s^{\prime}$ are scalar conductivities of $\mathcal{X}$ and $\mathcal{X}^{\prime}$ ?

Reconstruction. Assume that $(\mathcal{X}, \rho)$ is a two dimensional ohmic conductor. How from its DN-operator one can reconstruct a two dimensional ohmic conductor $\left(\mathcal{X}^{\prime}, \rho^{\prime}\right)$ which is isomorphic (in the above sense) to $(\mathcal{X}, \rho)$.

Characterization. Let $\gamma$ be a smooth abstract real curve, $L$ a complex line bundle along $\gamma$ and $\theta$ an operator from $C^{1}(\gamma)$ to the space of smooth sections of $L$. Find a non trivial necessary and sufficient condition on $(\gamma, L, \theta)$ which ensures that there exists a two dimensional ohmic conductor $(\mathcal{X}, \rho)$ such that $L=\Lambda^{1,0} T_{\gamma}^{*} \mathcal{X}$ and $\theta=\theta_{\mathcal{X}, \rho}$.

All these problems are open. In the particular case of constant scalar conductivity $\sigma$, the Dirichlet problem (2.7) becomes

$$
\left.U\right|_{\gamma}=u \& d d^{c} U=0
$$

where $d d^{c}=i \partial \bar{\partial}$ is the usual Laplacian. Hence, our article gives with theorems 1 to 3 a rather complete answer to the EIT-problem with constant scalar conductivity.

Concerning the main results given in the literature about unicity, reconstruction and stability for the important case where $\mathcal{X}$ is a domain in $\mathbb{R}^{2}$ but the scalar conductivity is not constant, see [8] 22] and references therein. Note that the exact method of reconstruction for this case goes back to [24].

\section{UNICITY UNDER EXISTENCE ASSUMPTION}

The notations and hypothesis are taken from theorem and section 1 ; we equip $\mathcal{X}$ with a hermitian metric ${ }^{(12)} \mathrm{g}$. Hence, there is a compact subset $\sigma$ of $\gamma$ such that $h^{1}(\sigma)=0$ and $(\overline{\mathcal{X}}, \gamma)$ is a manifold with boundary near each point of $\gamma \backslash \sigma$.

When $u \in C^{\infty}(\gamma)$, prop. 12 implies that $u$ has a continuous harmonic extension $\widetilde{u}$ with finite Dirichlet integral on $\mathcal{X}$. An elementary calculus gives then that for a fixed continuous defining function $\rho$ of $\gamma$, smooth on $\overline{\mathcal{X}} \backslash \sigma$, the operator $L$ defined by (1.1) determines for all $u \in C^{\infty}(\gamma)$ the trace on $\gamma$ of the holomorphic $(1,0)$-form $\partial \widetilde{u}: \partial \widetilde{u}=(L u)|\partial \rho|_{g}^{-1} \partial \rho$ on $\gamma \backslash \sigma$. With (1.2), this implies that $f$ is the restriction to $\gamma$ of a function $F=\left(F_{1}, F_{2}\right)$ meromorphic on $\mathcal{X}$, smooth on $\gamma \backslash \sigma$. Since $(\gamma, u, \theta u)$ is assumed to be a restricted DN-datum for $\mathcal{X}, F$ is continuous in a neighborhood of $\gamma$ in $\overline{\mathcal{X}}$.

The proof of theorem 1 relies on the following lemmas which enable to see $\overline{\mathcal{X}}$ as a normalization of $\overline{\mathcal{Y}}$.

Lemma 7. Set $\delta=f(\gamma)$. Then $\mathcal{Y}=F(\mathcal{X}) \backslash \delta$ is a complex curve of $\mathbb{C P}_{2} \backslash \delta$ without compact component, which has finite mass and satisfies $d[\mathcal{Y}]=[\delta]$. Moreover, each regular point of $\overline{\mathcal{X}}$ has in $\overline{\mathcal{X}}$ a neighborhood $V$ such that $F: V \rightarrow F(V)$ is diffeomorphism between manifolds with smooth boundary.

Proof. Since $F$ is continuous in a neighborhood of $\gamma$ in $\overline{\mathcal{X}}, \mathcal{Y}$ is a closed set of $\mathbb{C P}_{2} \backslash \delta$. As $\mathcal{Y}$ is also locally the image of a Riemann surface by an analytic map, $\mathcal{Y}$ is a complex curve of $\mathbb{C P}_{2} \backslash \delta$. Since $F_{*}[\mathcal{X}]$ is a locally flat

\footnotetext{
${ }^{12}$ Harmonicity does not depend of the chosen hermitian metric.
} 
current, the Federer support theorem (see [17, p. 316][14, 4.1.15 \& 4.1.20]) produces a locally integrable function $\lambda$ on $\mathcal{Y}$ such that $F_{*}[\mathcal{X}]=\lambda[\mathcal{Y}]$ on the regular part $\mathcal{Y}_{\text {reg }}$ of $\mathcal{Y}$; since $d^{2}=0, \lambda$ is locally constant. Since $f$ embeds $\gamma$ into $\mathbb{C}^{2}$, each point $x$ in $\gamma$ which is a regular boundary point of $\overline{\mathcal{X}}$ has a neighborhood $V$ of $x$ in $\overline{\mathcal{X}}$ such that $F: V_{x} \rightarrow F\left(V_{x}\right)$ is a diffeomorphism between classical manifolds with boundary. Let $F_{W}$ be the restriction of $F$ to the Riemann surfaces $W=\cup V_{x}$ and $W^{\prime}=F(W)$. The degree of $F_{W}$ is at most 1 otherwise almost all points of $W^{\prime}$ would have at least two different preimages which would imply that $d f$ is zero at almost all points of $\gamma$. So this degree is 1 and $F_{*}[\mathcal{X}]=[\mathcal{Y}]$ on $W^{\prime}$. Hence, $\lambda=1$ on each connected component of $\mathcal{Y}$ and $d[\mathcal{Y}]=[\delta]$.

If $\mathcal{Y}$ contains a compact complex curve $\mathcal{Z}, F^{-1}(\mathcal{Z})$ is a complex curve in $\mathcal{X}$ without boundary and so is empty. The fact that $\mathcal{Y}$ has a finite mass follows from a theorem of Wirtinger (see [17, Lemma 1.5 p. 315]).

As $\delta$ is smooth, the conclusion of lemma 0 implies, thanks to [18], that $\delta$ contains a compact set $\tau$ such that $h^{1}(\tau)=0$ and $(\overline{\mathcal{Y}}, \delta)$ is manifold with boundary near points of $\delta \backslash \tau$. The lemma below described how $\mathcal{Y}$ is near a point $y$ of $\tau$.

Lemma 8. Assume $\mathcal{Y}$ is a complex curve of $\mathbb{C P}_{2} \backslash \delta$ with finite mass satisfying $d[\mathcal{Y}]= \pm[\delta]$. Let $y$ be a point of $\sigma$ and $U$ a domain containing $y$. Then, among the components of $\mathcal{Y} \cap U, \mathcal{C}_{y, 1}^{U}, \ldots, \mathcal{C}_{y, m_{U}}^{U}$, one, says $\mathcal{C}_{y, 1}^{U}$, satisfies $d\left[\mathcal{C}_{y, 1}^{U}\right]= \pm\left.[\delta]\right|_{U_{y}}$ whereas for $j \geqslant 2, \overline{\mathcal{C}_{y, j}^{U}} \cap U$ is a complex curve of $U$.

Proof. 18, th. 4.7] implies that for each $j$ there is $n_{j} \in \mathbb{Z}$ such that $d\left[\mathcal{C}_{y, j}^{U}\right]=$ $n_{j}[\delta]$ on $U$. As $d[\mathcal{Y}]= \pm[\delta], \Sigma d\left[\mathcal{C}_{y, j}^{U}\right]= \pm 1$ and at least one $\mathcal{C}_{y, j}^{U}$, says $\mathcal{C}_{y, 1}^{U}$, is such that $n_{j} \neq 0$. Because $h^{1}(\sigma)=0, \delta \cap U$ contains a point $q$ not in $\sigma$. Then, if $V$ is a sufficiently small ball centered at $q, \mathcal{Y} \cap V$ is submanifold of $V$ with boundary $\delta \cap V$ and $\mathcal{Y} \cap V$ has only one connected component which can be nothing else than $\mathcal{C}_{y, 1}^{U} \cap V$. Hence $n_{1}= \pm 1$. Since two different bordered Riemann surfaces of some open set of $\mathbb{C P}_{2}$ meet at most in a set of zero one dimensional Hausdorff measure, this implies that $n_{j}=0$ for $j \neq 1$. Thus, if $j \geqslant 2, d\left[\mathcal{C}_{y, j}^{U}\right]=0$ and with [17, th. 2.1 p. 337] we conclude that $\overline{\mathcal{C}_{y, j}^{U}} \cap U$ is a complex curve of $U_{y}$.

If $y \in \delta$, we denote by $m_{y}$ the limit of $m_{U}$ (see lemma 8 ) when the diameter of $U$ goes to $0, U$ neighborhood of $y$; if $m_{y} \geqslant 2$, then $y \in \tau$. A point $y$ of $\delta$ is called a strong singularity of $\overline{\mathcal{Y}}$ if $y$ is not a regular point of $\mathcal{C}_{y, 1}^{U}$ and a weak singularity of $\overline{\mathcal{Y}}$ if $y$ is regular point of $\mathcal{C}_{y, 1}^{U}$ but $m_{y} \geqslant 2$.

We denote by $\tau_{1}$ (resp. $\tau_{2}$ ) the sets of points where $\overline{\mathcal{Y}}$ has weak (resp. strong) singularity. Then $\tau=\tau_{1} \cup \tau_{2}$ and $\tau_{1} \cap \tau_{2}=\varnothing$. Note that both $\tau_{1}$ and $\tau_{2}$ may contains points $y$ where $m_{y} \geqslant 2$.

We denote by $\overline{\mathcal{Y}}_{\text {sing }}=\mathcal{Y}_{\text {sing }} \cup \tau$ the singular locus of $\overline{\mathcal{Y}}$, that is the set of points of $\overline{\mathcal{Y}}$ where $\overline{\mathcal{Y}}$ is not a smooth manifold with boundary and we set $\mathcal{B}=f(\sigma) \cup \overline{\mathcal{Y}}_{\text {sing }}, \mathcal{A}=F^{-1}(\mathcal{B})$ and $\mathcal{X}_{\circ}=\mathcal{X} \backslash F^{-1}(\delta)=\mathcal{X} \backslash F^{-1}\left(\tau_{2}\right)$. 
Lemma 9. The map $F: \overline{\mathcal{X}} \rightarrow \overline{\mathcal{Y}}$ is a normalization in the following sense: $F: \mathcal{X}_{\circ} \rightarrow \mathcal{Y}$ is a (usual) normalization and $F: \overline{\mathcal{X}} \backslash \mathcal{A} \rightarrow \overline{\mathcal{Y}} \backslash \mathcal{B}$ is a diffeomorphism between manifolds with boundary.

Proof. Since $\mathcal{X}_{\circ}=\mathcal{X} \backslash F^{-1}(\delta)$, the properness of $\left.F\right|_{\mathcal{X}_{\circ}}$ and the finiteness of its fibers are elementary. For each connected component $\mathcal{C}$ of $\mathcal{X}_{\circ} \backslash \mathcal{A}$, the degree $m_{\mathcal{C}}$ of $F: \mathcal{C} \rightarrow F(\mathcal{C})$ as a Riemann surfaces morphism is finite and $F_{*}[\mathcal{C}]=\delta_{\mathcal{C}}[F(\mathcal{C})]$. Reasoning as in lemma 7 s proof, we get $m_{\mathcal{C}}=1$. As $\mathcal{Y}_{\text {sing }}$ contains all the points of $\mathcal{Y}$ which has more than one preimage by $F, F: \mathcal{C} \rightarrow F(\mathcal{C})$ is thus an isomorphism. Let $\mathcal{C}^{\prime}$ be another connected component of $\mathcal{X}_{\circ} \backslash \mathcal{A}$ and assume that $F(\mathcal{C})$ and $F\left(\mathcal{C}^{\prime}\right)$ meet at $q$. Since $q \notin \mathcal{B}$, the germs of $F(\mathcal{C})$ and $F\left(\mathcal{C}^{\prime}\right)$ at $q$ are equal. This leads to $F(\mathcal{C})=F\left(\mathcal{C}^{\prime}\right)$ which yields the contradiction $d[\mathcal{Y}]=2[\delta]$ near regular boundary points of $\delta$ in $b F(\mathcal{C})$. Hence, $F: \mathcal{X}_{\circ} \backslash \mathcal{A} \rightarrow \mathcal{Y} \backslash \mathcal{B}=\mathcal{Y}_{\text {reg }}$ is an isomorphism of complex manifolds. As $\mathcal{X}_{\circ} \cap \mathcal{A}=F^{-1}\left(\mathcal{Y}_{\text {sing }}\right)$ has empty interior, $F: \mathcal{X}_{\circ} \rightarrow \mathcal{Y}$ is a usual normalization.

Set $\widetilde{\mathcal{X}}=\overline{\mathcal{X}} \backslash \mathcal{A}, \widetilde{\mathcal{Y}}=\overline{\mathcal{Y}} \backslash \mathcal{B}, \widetilde{\tau}=\tau \cup f(\sigma)$ and $\widetilde{\delta}=\delta \backslash \widetilde{\tau}$; by definition of $\mathcal{B}, \widetilde{\mathcal{Y}}$ is a manifold with smooth boundary $\widetilde{\delta}=\delta \backslash \widetilde{\tau}$ and $\widetilde{\mathcal{X}}$ has smooth boundary $\gamma \backslash \widetilde{\sigma}$ where $\widetilde{\sigma}=f^{-1}(\tau)=\sigma \cup f^{-1}(\tau)$. The map $F: \widetilde{\mathcal{X}} \rightarrow \widetilde{\mathcal{Y}}$ is onto by construction. It is injective because the maps $F: \mathcal{X}_{\circ} \rightarrow \mathcal{Y}$ and $f: \gamma \rightarrow \delta$ are so and because if $x_{1} \in \mathcal{X}$ and $x_{2} \in \gamma$ have the same image $y$ by $F$, then $m_{y} \geqslant 2, y \in \tau$ and $x_{1}, x_{2} \in \mathcal{A}$. Since $\widetilde{\mathcal{Y}} \backslash \widetilde{\tau}=\mathcal{Y}_{\text {reg }}$ and $F: \mathcal{X}_{\circ} \backslash \mathcal{A} \rightarrow \mathcal{Y}_{\text {reg }}$ is a diffeomorphism, the fact that $F: \widetilde{\mathcal{X}} \rightarrow \widetilde{\mathcal{Y}}$ is a diffeomorphism between manifolds with boundary has only to be check locally near boundary points. If $x \in \gamma \backslash \tilde{\sigma}$, then $y=f(x) \notin \tau$ and the last conclusion of lemma 0 implies that there are open neighborhoods $V$ and $W$ of $x$ and $y$ in $\overline{\mathcal{X}}$ and $\overline{\mathcal{Y}}$ such that $F: V \rightarrow W$ is diffeomorphism between manifolds with boundary.

3.1. Proof of theorem 1. Let $L^{\prime}$ be the operator defined by (1.1) when $N$ is changed for $N^{\prime}$, let us denote $F^{\prime}$ the meromorphic extension of $f$ to $\mathcal{X}^{\prime}$ and let $\mathcal{Y}^{\prime}=F^{\prime}\left(\mathcal{X}^{\prime}\right) \backslash \delta$ where $\delta=f(\gamma)$. By lemma 7 , the sets $\mathcal{Y}^{\prime}$ and $\mathcal{Y}$ are two complex curves of $\mathbb{C P}_{2} \backslash \delta$ which has no compact component and both are bordered by $[\delta]$ in the sense of currents. Hence they are identical by a consequence of a Harvey-Shiffman theorem (see [12, prop. 1.4.1]).

Taking in account lemma 9 and the fact that $\mathcal{B} \cap \mathcal{Y}=\mathcal{Y}_{\text {sing }}=\mathcal{B}^{\prime} \cap$ $\mathcal{Y}$, this implies that $\Phi=F^{-1} \circ F^{\prime}$ is an analytic isomorphism between $\mathcal{X}^{\prime} \backslash F^{\prime-1}\left(\mathcal{Y}_{\text {sing }}\right)$ and $\mathcal{X}^{\prime} \backslash F^{-1}\left(\mathcal{Y}_{\text {sing }}\right)$. Using the properness of $F: \mathcal{X}_{\circ} \rightarrow \mathcal{Y}$ and $F^{\prime}: \mathcal{X}_{\circ}^{\prime} \rightarrow \mathcal{Y}$, we conclude that $\Phi$ extends holomorphically to $\mathcal{X}^{\prime}$. Likewise, $\Psi=F^{\prime-1} \circ F$ extends holomorphically to $\mathcal{X}$. As $\Phi\left(\Psi\left(x^{\prime}\right)\right)=x^{\prime}$ and $\Psi(\Phi(x))=x$ for almost all $x^{\prime} \in \mathcal{X}^{\prime}$ and $x \in \mathcal{X}$, the extension of $\Phi$ is an isomorphism from $\mathcal{X}$ to $\mathcal{X}^{\prime}$.

As $F$ and $F^{\prime}$ extend $f$ to $\mathcal{X}$ and $\mathcal{X}^{\prime}, \Phi$ extend continuously to $\gamma$ by the identity map on $\gamma$. Set $\sigma=\overline{\mathcal{X}}_{\text {sing }}$ and $\sigma^{\prime}=\overline{\mathcal{X}}_{\text {sing }}$ and let $x^{\prime}$ be in $\gamma \backslash\left(\sigma \cup \sigma^{\prime}\right)$. Then if $y=f(x) \notin \tau=\delta \cap \overline{\mathcal{Y}}_{\text {sing }}, \Phi$ is a diffeomorphism between neighborhoods of $x$ in $\overline{\mathcal{X}}$ and $\overline{\mathcal{X}^{\prime}}$ because $F$ (resp. $F^{\prime}$ ) is a diffeomorphism between manifold with boundary from a neighborhood of $x$ in $\overline{\mathcal{X}}$ (resp. in $\overline{\mathcal{X}^{\prime}}$ ) to a neighborhood of $y$ in $\overline{\mathcal{Y}}$. If $y \in \tau$, then the last conclusion of lemma 7 implies that $y \in \tau_{1}$ so that there is a open neighborhood $U$ of $y$, a component $\mathcal{C}_{y, 1}^{U}$ of $\mathcal{Y} \cap U$ and open neighborhoods $V$ and $V^{\prime}$ of 
$x$ in $\overline{\mathcal{X}}$ and $\overline{\mathcal{X}^{\prime}}$ such that that $F: V \rightarrow \mathcal{C}_{y, 1}^{U}$ and $F^{\prime}: V^{\prime} \rightarrow \mathcal{C}_{y, 1}^{U}$ are diffeomorphism between manifolds with smooth boundary. Hence, $\Phi: V \rightarrow$ $V^{\prime}$ is diffeomorphism between manifolds with smooth boundary. Finally, $\Phi$ realizes a diffeomorphism between manifolds with smooth boundary from $\mathcal{X}^{\prime} \backslash\left(\sigma \cup \sigma^{\prime}\right)$ to $\mathcal{X} \backslash\left(\sigma \cup \sigma^{\prime}\right)$ and the proof is complete.

The proof contains the following variation of theorem $\mathbb{1}$.

Theorem 10. Assume that $\mathcal{X}$ and $\mathcal{X}^{\prime}$ are open Riemann surfaces with almost smooth boundary $\gamma$ such that the map $f$ defined by (1.9) is an embedding of $\gamma$ into $\mathbb{C P}_{2}$ and has a meromorphic extension $F$ to $\mathcal{X}$ and $F^{\prime}$ to $\mathcal{X}^{\prime}$ which are continuous near $\gamma$. Then $F(\mathcal{X}) \backslash f(\gamma)=F^{\prime}\left(\mathcal{X}^{\prime}\right) / f(\gamma) \stackrel{\text { def }}{=} \mathcal{Y}$ is a complex curve of $\mathbb{C P}_{2} \backslash \delta$ without compact component, which has finite mass and satisfies $d[\mathcal{Y}]=[\delta]$. Moreover, $\overline{\mathcal{X}}$ and $\overline{\mathcal{X}^{\prime}}$ are normalizations of $\overline{\mathcal{Y}}$ in the sense of lemma 9 .

Thus, Riemann surfaces constructed in the converse part of theorems 3a, $3 \mathrm{~b}$ and $3 \mathrm{~d}$ are the only possible candidates for a solution to the IDN-problem.

\section{Existence And Reconstruction, proof of theorem 2}

We first prove that the Stokes formula holds in almost smoothly bordered manifolds; one can see also [14].

Lemma 11. Let $(\overline{\mathcal{X}}, \gamma)$ be a Riemann surface with almost smooth boundary. Then for any 1-form $\varphi$ which is continuous on $\overline{\mathcal{X}}$ such that $d \varphi$ exists as an integrable differential on $\mathcal{X}$, we have

$$
\int_{\mathcal{X}} d \varphi=\int_{\gamma} \varphi
$$

Proof. Set $\sigma=\overline{\mathcal{X}}_{\text {sing. Since }} h^{2}(\overline{\mathcal{X}})<\infty$ and $h^{1}(\sigma)=0$, there is an increasing sequence $\left(\mathcal{X}_{k}\right)$ of smooth open sets of $\mathcal{X}$ such that $\left(h^{1}\left(b \mathcal{X}_{k}\right)\right)$ and $\left(h^{2}\left(\overline{\mathcal{X}} \backslash \mathcal{X}_{k}\right)\right)$ both have limit zero and $\overline{\mathcal{X}} \backslash \overline{\mathcal{X}_{k}}$ is contained in a $2^{-k}$ neighborhood of $\sigma$. Let $\varphi$ be as above. Since $d \varphi$ is integrable and $\lim h^{2}\left(\mathcal{X}_{k}\right)=$ $0,\left(\int_{\mathcal{X}_{k}} d \varphi\right)$ has $\operatorname{limit} \int_{\mathcal{X}} d \varphi$. As $b \mathcal{X}_{k}=\left(\gamma \cap \overline{\mathcal{X}_{k}}\right) \cup\left[\left(b \mathcal{X}_{k}\right) \backslash \gamma\right]$ and $\lim h^{1}\left(b \mathcal{X}_{k}\right)=$ $0,\left(\int_{\left(b \mathcal{X}_{k}\right) \backslash \gamma} \varphi\right)$ converges to 0 . Hence, $\lim \int_{\gamma \cap \overline{\mathcal{X}_{k}}} \varphi=\int_{\gamma} \varphi$ and the classical Stokes formula for $\varphi$ and $\mathcal{X}_{k}$ yields (4.1).

We prove now a variation of the Riemann's existence theorem.

Proposition 12. Let $(\overline{\mathcal{X}}, \gamma)$ be a Riemann surface with almost smooth boundary and $u$ a real valued lipschitzian function on $\gamma$. Then $u$ has a unique continuous harmonic extension $\widetilde{u}$ of $u$ to $\mathcal{X}$ and $\widetilde{u}$ has finite Dirichlet integral $\int i \partial \widetilde{u} \wedge \bar{\partial} \widetilde{u}$. Moreover, $N_{\mathcal{X}} u$ defined as $\partial \widetilde{u} / \partial \nu$ on $\gamma \backslash \overline{\mathcal{X}}_{\text {sing }}$ admits an extension on $\gamma$ as a current of order 1 on $\gamma$.

Proof. Following the lines of Riemann's method for harmonic extension of smooth functions, we first construct an adequate space $W^{1}(\mathcal{X})$.

Since $(\overline{\mathcal{X}}, \gamma)$ is at least a topological bordered manifold, for every fixed point $x$ in $\gamma$ we can choose in $\mathcal{X}$ an open set $\Delta_{x}$ whose closure in $\overline{\mathcal{X}}$ is a neighborhood of $x$ and which is mapped by a complex coordinate $\varphi_{x}$ into 
the closure of the unit disk $\mathbb{D}$ of $\mathbb{C}, \varphi_{x}$ being a homeomorphism from $\overline{\Delta_{x}}$ to $\overline{\mathbb{D}}$. Note that if $x^{\prime} \in \gamma \cap \overline{\Delta_{x}}$ is a regular point of $\overline{\mathcal{X}}, \varphi_{x}$ has to be diffeomorphism between manifolds with boundary from a neighborhood of $x^{\prime}$ to a neighborhood of $\varphi_{x}\left(x^{\prime}\right)$ in $\overline{\mathbb{D}}$. If $x \in \mathcal{X}$, we choose a conformal open disk $\varphi_{x}: \Delta_{x} \rightarrow \mathbb{D}$ of $\mathcal{X}$ centered at $x$. With the help of a continuous partition of unity, we can now construct a continuous hermitian metric $h$ on $\mathcal{X}$ by gluing together the local metrics $\left(\varphi_{x}\right)_{*} d z \wedge d \bar{z}$ where $z$ is the standard coordinate of $\mathbb{C}$. We then denote by $W^{1}(\mathcal{X})$ the Sobolev space of functions in $L^{2}(\mathcal{X}, h)$ with finite Dirichlet integral.

By construction, any function $A$ in $W^{1}(\mathcal{X})$ is such that for each $x \in \gamma$, $B_{x}=\left.\left(\varphi_{x}\right)_{*} A\right|_{\Delta_{x}}$ is square integrable for the standard metric of $\mathbb{D}$. Since the values of Dirichlet integrals are conformal invariants, it follows that $B_{x}$ is in the standard Sobolev space $W^{1}(\mathbb{D})$ and hence admits a boundary value $b_{x}$ on $\mathbb{T}=b \mathbb{D}$ which is in $W^{1 / 2}(\mathbb{T})$. As $b_{x}$ is punctually defined almost everywhere, $a_{x}=b_{x} \circ \varphi$ is defined almost everywhere in $\gamma \cap \overline{\Delta_{x}}$. The constructions made for each $x \in \gamma$ glue together to form a function defined almost everywhere in $\gamma$ which we call the boundary value of $A$.

We consider now the subset $F$ of $W^{1}(\mathcal{X})$ with boundary value $u$. It is closed and non empty since by a result of McShane [23], $u$ admits a Lipschitz extension to $\mathcal{X}$. It follows now from classical arguments that the Dirichlet integral can be minimized in $F$ at some function $\widetilde{u}$ which has to be harmonic in $\mathcal{X}$. It remains only to show that $\widetilde{u}$ is continuous on $\overline{\mathcal{X}}$. If $x \in \gamma$, what precedes implies that $v_{x}=\left.\left(u_{x} \circ \varphi_{x}^{-1}\right)\right|_{\mathbb{T}}$ is in $W^{1 / 2}(\mathbb{T})$, continuous near $\varphi_{x}(x)$ and is the boundary value of $\widetilde{v}_{x}=\widetilde{u} \circ \varphi_{x}^{-1}$. Hence, classical Poisson formula for the disc implies that near $\varphi_{x}(x)$ in $\overline{\mathbb{D}}, \widetilde{v}_{x}$ is continuous up to $\mathbb{T}$ with restriction $v_{x}$ on $\mathbb{T}$. Since $\varphi_{x}$ is an homeomorphism, we get that $\widetilde{u}$ is continuous at $x$ with value $u(x)$.

Let $\theta u$ be the form defined by 1.1. The Stokes formula (4.1) implies that if $\varphi \in C^{1}(\gamma)$ and $\Phi$ is a Lipschitz extension of $\varphi$ on $\mathcal{X}$,

$$
\int_{\gamma} \varphi \theta u=-\int_{\mathcal{X}} \partial \widetilde{u} \wedge \bar{\partial} \Phi
$$

As the last integral is independent of the Lipschitz extension of $\varphi$, this means that $\theta u$ and hence $N_{\mathcal{X}} u$, are well defined currents of order 1 .

Assume now that the hypothesis of theorem 2 are true. Lemma 01 points out that $F$ projects $\gamma$ on a smooth curve $\delta$ of $\mathbb{C}^{2}$ which bounds in the sense of currents a complex curve $\mathcal{Y}$ of $\mathbb{C P}_{2} \backslash \delta$ which has finite mass and no compact component and theorem 10 implies that for some subset $\mathcal{X}_{\circ}$ of $\mathcal{X}$ with discrete complement in $\mathcal{X}, F: \mathcal{X}_{\circ} \rightarrow \mathcal{Y}$ is usual normalization. Hence, if $\mathcal{B}=\mathcal{Y}_{\text {sing }}$ and $\mathcal{A}=F^{-1}(\mathcal{B}), F: \mathcal{X} \backslash \mathcal{A} \rightarrow \mathcal{Y} \backslash \mathcal{B}$ is one to one. This is part 1 of theorem 2 .

Before proving the second claim of theorem 2, we recall that $\mathbb{C P}_{2}$ is equipped with homogenous coordinates $w$ and $\mathbb{C}^{2}$ identified with $\left\{w_{0} \neq 0\right\}$ has affine coordinates $z_{1}=w_{1} / w_{0}$ and $z_{2}=w_{2} / w_{0}$. Set $\Delta_{\infty}=\left\{w_{0}=0\right\}$, $\mathcal{Y}_{\infty}=\mathcal{Y} \cap \Delta_{\infty}$ and if $\xi \in \mathbb{C}$, we set $\Delta_{\xi}=\left\{w_{2}=\xi w_{0}\right\}$ and $\mathcal{Y}_{\xi}=\mathcal{Y} \cap \Delta_{\xi}$. Set

$$
\Omega_{\xi}^{m}=\frac{z_{1}^{m}}{z_{2}-\xi} d z_{2}=\frac{w_{1}^{m}}{w_{0}^{m}} \frac{d w_{2}}{w_{2}-\xi w_{0}}-\frac{w_{1}^{m}}{w_{0}^{m+1}} \frac{w_{2} d w_{0}}{w_{2}-\xi w_{0}} .
$$


Applying the Stokes formula either for $\mathcal{Y}$ or $\mathcal{X}$, it turns out that $\frac{1}{2 \pi i} \int_{\gamma} \frac{f_{1}^{m}}{f_{2}-\xi} d f_{2}$ equals $S_{m}(\xi)+P_{m}(\xi)$ where

$$
S_{m}(\xi)=\sum_{z \in \mathcal{Y}_{\xi}} \operatorname{Res}\left(\eta^{*} \Omega_{\xi}^{m}, z\right) ; P_{m}(\xi)=\sum_{z \in \mathcal{Y}_{\infty}} \operatorname{Res}\left(\eta^{*} \Omega_{\xi}^{m}, z\right)
$$

and $\eta: \mathcal{Y} \rightarrow \mathbb{C P}_{2}$ is the canonical injection.

For almost all $\xi_{*}$ in $\mathbb{C}, \mathcal{Y}$ meets transversely $\Delta_{\xi_{*}}$ only in $\mathbb{C}^{2} \cap \mathcal{Y}_{\text {reg }}$; for such a fixed $\xi_{*}$, set $p=\operatorname{Card} \mathcal{Y}_{\xi_{*}}$ and $\mathcal{Y}_{\xi_{*}}=\left\{z_{1 *}, \ldots, z_{p *}\right\}$. For $\xi$ in a sufficiently small connected neighborhood $W_{\xi_{*}}$ of $\xi_{*}, \mathcal{Y}_{\xi}$ lies then in $\mathbb{C}^{2} \cap \mathcal{Y}_{\text {reg }}$ and can be written $\left\{z_{1}(\xi), \ldots, z_{p}(\xi)\right\}$ with $z_{j}(\xi)=\left(h_{j}(\xi), \xi\right)$ where $h_{j}$ is holomorphic in $W_{\xi_{*}}$ and has value $z_{j *}$ at $\xi_{*}, 1 \leqslant j \leqslant p$. Direct calculation shows (see [12]) that the poles of $\Omega_{\xi}^{m}$ in $\mathbb{C}^{2}$ are $z_{1}(\xi), \ldots, z_{p}(\xi)$ with residue $h_{1}(\xi)^{m}, \ldots, h_{p}(\xi)^{m}$. Hence, $S_{m}=S_{h, m}$ in $V$.

Reasoning as in lemma 13 in next section, we can assume without loss of generality that $\mathcal{Y}$ meets transversely $\Delta_{\infty}$ and that $\mathcal{Y}_{\infty} \subset \mathcal{Y}_{\text {reg. }}$. In this situation, a direct calculus (see [12]) gives that at $y \in \mathcal{Y}_{\infty}, \Omega_{\xi}^{m}$ has a pole of order $m+1$ with a residue which is a polynomial in $\xi$ of degree at most $m$. Hence, $P_{m}$ is is a polynomial in $\xi$ of degree at most $m$; formula $\left(E_{m . g}\right)$ is proved.

If $A \geqslant B$ and $\xi_{0}, \ldots, \xi_{A-1}$ are mutually distinct, the Vandermonde matrix $\left(\xi_{\nu}^{\mu}\right)_{0 \leqslant \nu, \mu \leqslant B-1}$ is invertible and hence, the system $\left(E_{m, \xi_{\nu}}\right)_{0 \leqslant \nu \leqslant B-1}$ enable to write the coefficients of $P_{m}$ as a linear combination of the $S_{h, m}\left(\xi_{\nu}\right)$, $0 \leqslant \nu \leqslant B-1$. Introducing this result in $\left(E_{\xi}\right)=\left(E_{m, \xi_{\nu}}\right)_{\substack{0 \leqslant m \leqslant B-1 \\ 0 \leqslant \nu \leqslant B-1}}$ we get a linear system which, since $A B-\frac{1}{2} B(B+1) \geqslant p A$ when $B \geqslant 2 p+1$, enable to compute for a generic $\xi$ the unknowns $S_{h, m}\left(\xi_{\nu}\right)$ and, thanks to the NewtonGirard formulas, the elementary symmetric functions of $h_{1}\left(\xi_{\nu}\right), \ldots, h_{p}\left(\xi_{\nu}\right)$; finally we get the intersection points $\left(h_{j}\left(\xi_{\nu}\right), \xi_{\nu}\right)$ of $\mathcal{Y}$ with $\Delta_{\xi_{\nu}}$.

We prove the third assertion of theorem 2. Almost all $\xi_{*}$ in $\mathbb{C}^{2}$ has a connected neighborhood $W_{\xi_{*}}$ such that there is a compact of $\mathbb{C P}_{2} \backslash\left(\delta \cup \mathcal{Y}_{\text {sing }}\right)$ containing all $\mathcal{Y}_{\xi}$ when $\xi \in W_{\xi_{*}}$. When $\xi_{*}$ is such and $\xi \in W_{\xi_{*}}$ the form $\Phi_{\xi}^{m, \ell}=\frac{F_{1}^{m}}{F_{2}-\xi} \partial \widetilde{u_{\ell}}$ may have poles of order at most $m$ at infinity i.e. in $\left\{w_{0}=0\right\} \cap \mathcal{X}$ and whiles its over poles lies in a compact of $\mathcal{X}$. Since $\widetilde{u}_{\ell}$ is the continuous harmonic extension of $u_{\ell}$ on $\mathcal{X}, \int_{\mathcal{X}} i \partial \widetilde{u}_{\ell} \wedge \bar{\partial} \widetilde{u}_{\ell}<+\infty$ by proposition 12 and we can apply the Stokes formula (4.1) to it on $\mathcal{X}$. This gives $\left(T_{m, \xi}\right)$ after a residue calculus.

Remark. The $\frac{1}{2} B(B+1)$ coefficients of the polynomials $P_{k}$ come from the residues of the intersection points of $\mathcal{Y}$ with $\left\{w_{0}=0\right\}$. In the generic case, $\mathcal{Y}$ is given near theses points as the graph of holomorphic functions $\psi_{1}, \ldots, \psi_{q}$ of the variable $w_{0} / w_{2}$, it appears that the coefficients of $P_{k}$ are ruled by the derivatives of order at most $k$ at 0 of the $\psi_{\ell}$. The reconstruction of $\mathcal{X}$ is thus possible with a non linear system with only $p A+q(B+1)$ unknowns.

\section{Proofs of characterizations theorem 3A, 3B and 3a}

The proofs of theorems $3 \mathrm{a}$, $3 \mathrm{~b}$ and $3 \mathrm{c}$ follow a similar schema. The function $f$ defined by (1.2) embeds $\gamma$ into a smooth real curve $\delta=f(\gamma)$ of $\mathbb{C}^{2}$. The necessary conditions for the existence of a solution to the IDN-problem 
for $\gamma$ are drawn from the fact this existence implies that $\delta$ bounds a "concrete" Riemann surface in $\mathbb{C P}_{2}$ or $\mathbb{C}^{2}$. The sufficient part of theorem $3 \mathrm{Bc}$ reconstructs the concrete but singular solution to the IDN-problem ; a normalization gives then the sufficient part of $3 \mathrm{~b}$. The proof of theorem $3 \mathrm{a}$ follows a similar scheme.

5.1. Proof of A.theorem 3a. Assume that $\mathcal{X}$ is an open bordered riemannian surface of finite volume with restricted DN-datum $(\gamma, u, \theta u)$. Then the functions $F_{j}(j=1,2)$ which are the well defined quotients of forms $\left(\partial \widetilde{u_{j}}\right) /\left(\partial \widetilde{u}_{0}\right)$ are meromorphic and letting $F=\left(F_{1}, F_{2}\right)$, lemma 0 implies that $\mathcal{Y}=F(\mathcal{X}) \backslash \delta, \delta=f(\gamma)$, is a complex curve of finite volume, without compact component and bordered by $[\delta]$ in the sense of currents. Moreover, the function $G$ has the expression

$$
G\left(\xi_{0}, \xi_{1}\right)=\frac{1}{2 \pi i} \int_{\delta} \Omega_{\xi}, \Omega_{\xi}=\frac{w_{1}}{w_{0}} \frac{d \Lambda_{\xi}(w)}{\Lambda_{\xi}(w)}-\frac{w_{1}}{w_{0}^{2}} d w_{0}
$$

where $\delta=f(\gamma),\left(w_{0}: w_{1}: w_{2}\right)$ are homogenous coordinates for $\mathbb{C P}_{2}$ and $\Lambda_{\xi}(w)=\xi_{0} w_{0}+\xi_{1} w_{1}+w_{2}$.

For almost all $\xi_{*}=\left(\xi_{0 *}, \xi_{1 *}\right)$ and for all $\xi$ in a sufficiently small connected neighborhood $W_{\xi_{*}}$ of $\xi_{*}, \mathcal{Y}$ meets $\Delta_{\xi}=\left\{\Lambda_{\xi}=0\right\}$ transversely, $\mathcal{Y}_{\xi}=\mathcal{Y} \cap$ $\Delta_{\xi} \subset \mathbb{C}^{2} \cap \mathcal{Y}_{\text {reg }}$ so that there exists $p=$ Card $\mathcal{Y}_{\xi_{*}}$ holomorphic functions $H_{j}=\left(1: h_{j}: h_{j, 2}\right): W_{\xi_{*}} \rightarrow \mathbb{C P}_{2}$ such that $\mathcal{Y}_{\xi}=\left\{H_{j}(\xi), 1 \leqslant j \leqslant p\right\}$ and $\left(h_{j}\right)_{1 \leqslant j \leqslant p}$ are mutually distinct. Direct calculations ${ }^{(13)}$ shows that these functions satisfy the shock wave equation (1.5).

Let $\eta: \mathcal{Y} \rightarrow \mathbb{C P}_{2}$ the canonical injection. Since $\eta^{*} \Omega_{\xi}$ may only have poles in $\mathcal{Y}_{\xi} \cup \mathcal{Y}_{\infty}$, the Stokes formula gives that near $\xi_{*}, G=H+L$ where

$$
H(\xi)=\sum_{z \in \mathcal{Y}_{\xi}} \operatorname{Res}\left(\eta^{*} \Omega_{\xi}, z\right) \quad, \quad L(\xi)=\sum_{z \in \mathcal{Y}_{\infty}} \operatorname{Res}\left(\eta^{*} \Omega_{\xi}, z\right) .
$$

By construction, $\eta^{*} \Omega_{\xi}$ has residue $h_{j}(\xi)$ at $z=H_{j}(\xi) \in \mathcal{Y}_{\xi}$ and it remains only to know that $L$ is affine in $\xi_{0}$ to prove theorem 3 . The second part of the lemma below is needed in the proof of theorem 4 .

Lemma 13. If $W_{\xi_{*}}$ is small enough, $L=\Sigma h_{j}-G$ is affine in $\xi_{0}$. In addition, there is an integer $q$ such that $L$ is the limit in $\mathcal{O}\left(W_{\xi^{*}}\right)$ of a continuous one parameter family of $\xi_{0}$-affine functions which are sum of $q$ mutually distinct shock wave functions.

Proof. With no loss of generality, we assume $\xi_{*}=0$ for the proof. For small complex parameters $\varepsilon$, we consider the homogeneous coordinates $w^{\varepsilon}=$ $\left(w_{0}+\varepsilon w_{1}: w_{1}: w_{2}+\varepsilon w_{1}\right)$. For $\varepsilon$ in a sufficiently small neighborhood of 0 the intersection of $\mathcal{Y}$ with the zero set of $\Lambda_{\xi}: w \mapsto \xi_{0}+\xi_{1} w_{1}^{\varepsilon}+w_{2}^{\varepsilon}$ is still generic in the sense that it is transverse and lies in $\left\{w_{2}^{\varepsilon} \neq 0\right\} \cap \mathcal{Y}_{\text {reg. }}$. Hence, setting $\Omega_{\xi}^{\varepsilon}=\frac{w_{1}}{w_{0}^{\varepsilon}} \frac{d \Lambda_{\xi}^{\varepsilon}(w)}{\Lambda_{\xi}^{\varepsilon}(w)}-\frac{w_{1}}{\left(w_{0}^{\varepsilon}\right)^{2}} d w_{0}^{\varepsilon}$, the function $G^{\varepsilon}: \xi \mapsto \frac{1}{2 \pi i} \int_{\delta} \Omega_{\xi}^{\varepsilon}$ is, on $W_{\xi_{*}}$ the sum of $p$ mutually distinct shock wave functions $h_{1}^{\varepsilon}, \ldots, h_{p}^{\varepsilon}$. For generic $\varepsilon$, $\mathcal{Y}$ meets transversely $\Delta_{\infty}^{\varepsilon}=\left\{w_{0}^{\varepsilon}=0\right\}$ and $\mathcal{Y}_{\infty}^{\varepsilon}=\mathcal{Y} \cap\left\{w_{0}=0\right\}$ lies in $\mathcal{Y}_{\text {reg }} \cap$ $\left\{w_{2}^{\varepsilon} \neq 0\right\}$. Hence, 12, Lemme 2.3.1] implies that $L^{\varepsilon}=\Sigma h_{j}^{\varepsilon}-G^{\varepsilon}$ is affine in $\xi_{0}$. The dependence of $G^{\varepsilon}$ is clearly holomorphic in $\varepsilon$. The same holds for

\footnotetext{
${ }^{13}$ This lemma which goes back to Darboux is proved in [12, lemma 2.4].
} 
each $h_{j}^{\varepsilon}$ since what precedes have shown that $h_{j}^{\varepsilon}(\xi)=\operatorname{Res}\left(\eta^{*} \Omega_{\xi}^{\varepsilon}, H_{j}(\xi)\right)=$ $\frac{1}{2 \pi i} \int_{\mathcal{Y} \cap \partial U_{j}} \eta^{*} \Omega_{\xi}^{\varepsilon}$ where $U_{j}$ is any sufficiently small neighborhood of $H_{j}(\xi)$ in $\mathbb{C P}_{2}$ whose boundary is smooth and transverse to $\mathcal{Y}$. Hence $L^{\varepsilon}$ is holomorphic in $\varepsilon$ and has to be affine in $\xi_{0}$ when $\varepsilon=0$.

Let $q$ be the number of points in $\mathcal{Y}_{\infty}$ counted with their multiplicities ; when $\xi$ is generic, $q$ is either defined by

$$
p-q=\frac{1}{2 \pi i} \int_{\gamma} \frac{d\left(\xi_{0}+\xi_{1} f_{1}+f_{2}\right)}{\xi_{0}+\xi_{1} f_{1}+f_{2}} .
$$

For sufficiently small generic $\varepsilon$, 12, Lemme 2.3.1] gives more precisely that $L^{\varepsilon}=\sum_{1 \leqslant j \leqslant q} h_{j}^{\varepsilon, \infty}$ with

$$
h_{j}^{\varepsilon, \infty}=-\operatorname{Res}\left(\eta^{*} \Omega_{\xi}, z_{j}^{\varepsilon}\right)=\frac{-\xi_{0} \psi_{j}^{\varepsilon}(0)+\psi_{j}^{\varepsilon \prime}(0)}{1+\xi_{1} \psi_{j}^{\varepsilon}(0)}
$$

where $\mathcal{Y}_{\infty}^{\varepsilon}=\left\{z_{1}^{\varepsilon}, \ldots, z_{q}^{\varepsilon}\right\}$ and $\psi_{j}^{\varepsilon} \in \mathcal{O}\left(U^{\varepsilon}\right), U^{\varepsilon}$ open neighborhood of 0 in $\mathbb{C}$, enable to give in the affine coordinates $\zeta^{\varepsilon}=\left(w_{j}^{\varepsilon} / w_{2}^{\varepsilon}\right)_{j=0,1}$ the set $\mathcal{Y}$ as a graph above $U^{\varepsilon}: \mathcal{Y} \cap V_{j}^{\varepsilon}=\left\{\left(\zeta_{0}^{\varepsilon}: \psi_{j}^{\varepsilon}\left(\zeta_{0}^{\varepsilon}\right): 1\right) ; \zeta_{0}^{\varepsilon} \in U^{\varepsilon}\right\}$. Each $h_{j}^{\varepsilon, \infty}$ is clearly a shock wave function, that is a solution to $h_{\xi_{1}}=h_{\xi_{0}} h$.

Remark. When $\varepsilon$ goes to a non generic value, the fact that $L$ is a sum of $q$ shock wave functions may not be preserved as section 6.1 shows.

5.2. Proof of B.theorem 3a. Assume that $\gamma$ satisfies (1.4) in a connected neighborhood $W_{\xi_{*}}$ of one point $\left(\xi_{0 *}: \xi_{1 *}: 1\right)$ of $\mathbb{C P}_{2}$. If $\left(\partial^{2} G / \partial \xi_{0}^{2}\right)_{\mid W_{\xi_{*}}}=0$, then $\gamma$ satisfies the classical Wermer-Harvey-Lawson moment condition in $\mathbb{C}_{\xi_{*}}^{2}=\mathbb{C P}_{2} \backslash\left\{\xi_{0 *} w_{0}+\xi_{1 *} w_{1}+w_{2}=0\right\}$ (see [12, cor. 1.6.2]) and [29] 18] implies that if $\delta$ is suitably oriented, the polynomial hull of $\delta$ in $\mathbb{C}_{\xi_{*}}^{2}$ is the unique complex curve $\mathcal{Y}$ of finite mass of $\mathbb{C}_{\xi_{*}}^{2} \backslash \delta$ such that $d[\mathcal{Y}]=[\delta]$.

Assume now $\left(\partial^{2} G / \partial \xi_{0}^{2}\right)_{\mid W_{\xi_{*}}} \neq 0$. Then we can choose a minimal $\mathcal{H}=$ $\left\{h_{1}, \ldots, h_{p}\right\}$ in the sense that no proper subset of $\mathcal{H}$ satisfy (1.4). Although it is not explicitly mentioned by their authors, the heart of the arguments of [12, th. II p. 390] is that $\pm[\delta]=d[\mathcal{Y}]$ where $\mathcal{Y}$ is the analytic extension $\mathcal{Y}$ in $\mathbb{C P}_{2} \backslash \delta$ of the union $\Gamma$ of the graphs $\Gamma_{j}$ of the functions

$$
H_{j}: \xi \mapsto\left(1: h_{j}(\xi):-\xi_{0}-\xi_{1} h_{j}(\xi)\right), 1 \leqslant j \leqslant p .
$$

This fact, not totally explicit in [20, p. 264], can be recovered a posteriori by a kind of trick which has been used Poly in [13] and is developed later in the proof of theorem $3 \mathrm{~g}$ : for the curve $\widetilde{\gamma}$ which is the union of $\gamma$ with the boundaries of $\Gamma_{j}$ negatively oriented, one goes back to the $\mathbb{C}_{\xi_{*}}^{2}$-case where $\left(\partial^{2} G / \partial \xi_{0}^{2}\right)_{W_{\xi_{*}}}=0$. If $d[\mathcal{Y}]$ is $-[\delta]$ and not $[\delta]$, then the same arguments which have proved theorem 3a. A would give that the functions $h_{j}$, geometrically defined as the first coordinates of points of intersection of $\mathcal{Y}$ with generic lines $\Lambda_{\xi}$, should satisfy not only the shock wave equation $h_{\xi_{1}}=h_{\xi_{0}} h$ but also the "negative" shock wave equation $h_{\xi_{1}}=-h_{\xi_{0}} h$. As this is impossible, $d[\mathcal{Y}]=[\delta]$. 
In both cases, we have found (up to a change of orientation if $\partial^{2} G / \partial \xi_{0}^{2}$ vanish on $\left.W_{\xi_{*}}\right)$ a complex curve $\mathcal{Y}$ of finite mass of $\mathbb{C P}_{2} \backslash \delta$ such that $d[\mathcal{Y}]=$ $[\delta]$.

As $\delta$ is smooth, we know from [18] that there is in $\delta$ a compact set $\tau$ such that $h^{1}(\tau)=0$ and for which each point of $y \in \delta \backslash \tau$ has a neighborhood $U_{y}$ where $\overline{\mathcal{Y}} \cap U_{y}$ is a closed bordered submanifold of $U_{y}$ with boundary $\delta \cap U_{y}$. Lemma 8 in section 3 describes how $\mathcal{Y}$ is near points of $\tau$. Using the notations and definitions introduced after its proof, we let $\tau_{2}$ (resp. $\tau^{\prime}$ ) be the set of $y$ in $\delta$ where $\overline{\mathcal{Y}}$ has a strong singularity (resp. $m_{y} \geqslant 2$ ) and define $\widetilde{\mathcal{Y}}$ as the abstract complex curve $\mathcal{Y} \cup \tau^{\prime}$.

Consider a normalization $\pi: \mathcal{X} \rightarrow \widetilde{\mathcal{Y}}$; lemma 8 implies that $\pi$ is an open mapping. Let $\mathcal{Z}$ be the disjoint and abstract union $\mathcal{X} \cup \gamma$. If $x \in \gamma$, we define a neighborhood of $x$ in $\mathcal{Z}$ as a subset of $\mathcal{Z}$ which contains a set of the kind $\pi^{-1}\left(C_{y, 1}^{U}\right)$ where $y=f(x)$ and $U$ is a neighborhood of $y$ in $\mathbb{C P}_{2}$. Then $(\mathcal{Z}, \gamma)$ is a compact metrizable topological manifold with boundary which has finite 2-dimensionnal Hausdorff measure and smooth boundary outside $\sigma=f^{-1}\left(\tau_{2}\right)$. Since $f$ is an embedding, $h^{1}(\sigma)=0$ and $(\mathcal{Z}, \gamma)$ is a manifold with almost smooth boundary. Moreover, it follows by construction that the meromorphic extension $F: \mathcal{Z} \rightarrow \overline{\mathcal{Y}}$ of $f$ to $\mathcal{X}$ defined by $\left.F\right|_{\mathcal{X}}=\pi$ is a normalization of $\overline{\mathcal{Y}}$ in the sense of lemma $9 \cdot \square$

Remark. When $\gamma$ is real analytic, [18, th. II] implies that $\mathcal{C}_{y, 1}^{U}$ is a manifold with boundary in the classical sense. So, in that case, $(\mathcal{Z}, \gamma)$ is a classical manifold with boundary.

The following proposition which clarifies some results of [12] justifies the fourth remark after theorem 3a.

Proposition 14. Assume $\delta$ is connected. Then if $\left(\partial^{2} G / \partial \xi_{0}^{2}\right)_{\mid W_{\xi_{*}}}=0$, the polynomial hull of $\gamma$ in $\mathbb{C}^{2}=\mathbb{C P}_{2} \backslash\left\{\xi_{0 *} w_{0}+\xi_{1 *} w_{1}+w_{2}=0\right\}$ has boundary $\pm[\gamma]$. If $\left(\partial^{2} G / \partial \xi_{0}^{2}\right)_{\mid W_{\xi_{*}}} \neq 0$ and no proper subset of $\mathcal{H}=\left\{h_{1}, \ldots, h_{p}\right\}$ satisfies (1.4), then the analytic extension $\mathcal{Y}$ in $\mathbb{C P}_{2} \backslash \delta$ of the union $\Gamma$ of the graphs $\Gamma_{j}$ of the functions $H_{j}: \xi \mapsto\left(1: h_{j}(\xi):-\xi_{0}-\xi_{1} h_{j}(\xi)\right), 1 \leqslant j \leqslant p$, is the complex curve which has minimal volume among complex curves $\mathcal{Z}$ such that $d[\mathcal{Z}]=[\delta]$.

Proof. The preceding proof contains the above conclusion except the minimality of volume of $\mathcal{Y}$ when $\left(\partial^{2} G / \partial \xi_{0}^{2}\right)_{\left.\right|_{W_{*}}} \neq 0$ and $\mathcal{H}$ is minimal. In that case, let $\mathcal{Y}^{\prime}$ be a complex curve of $\mathbb{C P}_{2} \backslash \delta$ with boundary $[\delta]$ and minimal volume. Then $\mathcal{Y}=\mathcal{Y}^{\prime} \cup \mathcal{Z}$ where $\mathcal{Z}$ is a union of compact complex curves of $\mathbb{C P}_{2}$. But the intersection of any compact curve with a line $\Lambda_{\xi}$ is described, for $\xi$ in a neighborhood of a generic $\xi_{*}$, as a finite union of graphs of function $\left(1: g_{j}: g_{j, 2}\right)$ whose second homogeneous coordinate satisfy the shock wave equation and such that $\Sigma g_{j}$ is affine in $\xi_{0}$ (see [20, section 2]). Since $\mathcal{H}$ is minimal, no such $g_{j}$ belongs to $\mathcal{H}$ and it appears that $\Gamma$ has to be contained in $\mathcal{Y}^{\prime}$ Hence, $\mathcal{Y} \subset \mathcal{Y}^{\prime}$ and, finally, $\mathcal{Y}=\mathcal{Y}^{\prime}$.

5.3. Proof of C.theorem 3a. Let $\mathcal{X}, \mathcal{Z}, \mathcal{D}$ and $g$ be as in the statement. Let $u \in C^{1}(\gamma)$ and for $z \in \mathcal{D} \backslash \gamma$ set

$$
\Omega_{z}=u \partial_{\zeta} g_{z}+g_{z} \overline{\theta u} \text {. }
$$


If $\mathcal{Y}$ is a smooth domain in $\mathcal{Z}$, the Stokes formula (4.1) implies that value of $\mathbf{1}_{\mathcal{Y}}(z) \widehat{u}(z)+\int_{\mathcal{Y}} \bar{\partial} \widehat{u} \wedge \partial_{\zeta} g_{z}-\int_{(\partial \mathcal{Y}) \backslash \gamma} u \partial_{\zeta} g_{z}$ does not depend of the Lipschitz extension $\widehat{u}$ of $u$ to $\mathcal{Z}$ and equals $\int_{\gamma \cap \mathcal{Y}} u \partial_{\zeta} g_{z}$ when $\mathcal{X}_{\text {sing }}=\varnothing$. Hence we can take it as a definition for $\int_{\gamma \cap \mathcal{Y}} u \partial_{\zeta} g_{z}$ in the general case. Let then $F$ be the function defined for $z \in \mathcal{D} \backslash \gamma$ by

$$
F(z)=\frac{2}{i} \int_{\zeta \in \gamma} \Omega_{z}(\zeta), \Omega_{z}=u \partial_{\zeta} g_{z}+g_{z} \overline{\theta u},
$$

where $g_{z}=g(., z)$.

Since $\widetilde{u}, u$ and $\theta u$ are continuous, the conclusion of part $\mathrm{C}$ follows from the lemma below which gives $\widetilde{u}=\left.F\right|_{\mathcal{X}}$ and $\theta u=\partial \widetilde{u}$ on $\gamma \backslash \sigma$ if $\left.F\right|_{\mathcal{D} \backslash \overline{\mathcal{X}}}=0$.

Lemma 15. $F_{+}=\left.F\right|_{\mathcal{X}}$ and $F_{-}=\left.F\right|_{\mathcal{D} \backslash \overline{\mathcal{X}}}$ are real valued harmonic functions such that

$$
u=F_{+}-F_{-} \& \overline{\theta u}=\bar{\partial} F_{+}-\bar{\partial} F_{-} \text {on } \gamma \backslash \sigma .
$$

Proof. The harmonicity of $F$ is a simple consequence of the properties of $g$. Fix now $p$ in $\gamma \backslash \sigma$ and in neighborhood $\mathcal{U}$ of $p$ in $\mathcal{D}$, a holomorphic chart $\mathcal{U} \rightarrow U$ centered at $p$; a hat " " denotes thereafter the coordinate expression of a function, a form or a set. For $z \in U \backslash \gamma$ let us write $F(z)=\varphi(z)+R_{1}(z)$ with $\varphi(z)=-2 i \int_{\gamma \cap \mathcal{U}} \Omega_{z}$ and $R_{1}$ is smooth on $\mathcal{U}$. If $y$ and $x$ are the coordinates of $\zeta \in \gamma \cap \mathcal{U}$ and $z \in U \backslash \gamma, \widehat{g}(y, x)=g(\zeta, z)$ can be written in the form $\widehat{g}(y, x)=\frac{1}{2 \pi} \ln |y-x|+h(y, x)$ where $h$ is a smooth function on $U \times U$, harmonic in each its variable. Hence

$$
\widehat{\varphi}(x)=\frac{1}{2 \pi i} \int_{\widehat{\gamma} \cap U} \frac{\widehat{u}(y)}{y-x} d y+\int_{\widehat{\gamma} \cap U} \frac{\ln |y-x|}{\pi i} \widehat{\widehat{\theta u}(y)}+R_{2}(x)
$$

where $R_{2} \in C^{0}(U)$. The second integral has no jump across $\widehat{\gamma}$ and from the classical Sohotsky-Plemelj formula, we know that the first integral has jump $\widehat{u}$ across $\widehat{\gamma}$ in the sense of distribution and pointwise near each regular boundary point. Since

$$
\bar{\partial} \widehat{\varphi}(x)=\frac{1}{2 \pi i} d \bar{x} \int_{\widehat{\gamma} \cap U} \frac{1}{\bar{x}-\bar{y}} \overline{\theta u}(y)+\bar{\partial} R_{2}(x),
$$

the jump of $\bar{\partial} \widehat{\varphi}$ through $\widehat{\gamma}$ is likewise $\overline{\widehat{\theta u}}$.

In order to check that $F(z) \in \mathbb{R}$ when $z \in \mathcal{D} \backslash \gamma$, we let $L_{\mathcal{X}}$ be the DNoperator of $\mathcal{X}$ and we note that since $\partial g_{z}=\left(L_{\mathcal{X}} g_{z}\right)\left(\nu^{*}+i \tau^{*}\right)$ and $\theta u=$ $(L u)\left(\nu^{*}+i \tau^{*}\right),-\operatorname{Im} F(z)=\int_{\gamma}\left(u \tau g_{z}+g_{z} \tau u\right) \tau^{*}=\int_{\gamma} d\left(\left.u g_{z}\right|_{\gamma}\right)=0$.

5.4. Proof of A.theorem 3d. We assume that $\gamma$ is in the sense of currents the boundary of a complex curve $\mathcal{X}$ of $\mathbb{C P}_{2} \backslash \gamma$ for which $(\gamma, u, \theta u)$ is a restricted DN-datum. Then as in the proof of theorem 3a. A, for almost all $\left(\xi_{* 0}: \xi_{* 1}: 1\right)$ in $\mathbb{C P}_{2}$ there exists a neighborhood $W_{\xi_{*}}$ of $\xi_{*}=\left(\xi_{* 0}, \xi_{* 1}\right)$ such that for every $\xi \in W_{\xi_{*}}, \mathcal{X} \cap \Delta_{\xi}$ lies in $\mathbb{C}^{2}$ and equals $\Gamma \cap \Delta_{\xi}$ where $\Gamma$ is the union of the graphs $\Gamma_{j}$ of $H_{j}=\left(1: h_{j}: h_{j, 2}\right): W_{\xi_{*}} \rightarrow \mathbb{C P}_{2}, 1 \leqslant j \leqslant p$ where $H_{j}$ is holomorphic in $W_{\xi_{*}}$. Since we are concerned only by generic $\xi_{*}$, we can suppose that $\Gamma_{j}=\left\{\left(\varphi_{j}\left(z_{2}\right), z_{2}\right) ; z_{2} \in U_{j}\right\}$ where $U_{j}$ is a neighborhood of $z_{j, 2}^{*}=h_{j, 2}\left(\xi_{*}\right)$ and $\varphi_{j} \in \mathcal{O}\left\{U_{j}\right\}$. The decomposition sought for $\widetilde{G}$ 
in (a1) can be then found in 20]. However, this residues calculus is needed in part $\mathrm{B}$ and we include it here

When $\xi \in W_{\xi_{*}}, \widetilde{G}_{\ell}\left(\xi_{0}: \xi_{1}: 1\right)$ is the sum of the residues of

$$
\Lambda_{\ell}=\frac{z_{\ell}}{\xi_{0}+\xi_{1} z_{1}+z_{2}} \Theta_{0}
$$

(for convenience $z_{\ell}=1$ if $\ell=0$ ) in $\mathcal{X}$. Set $\Theta_{0}=A_{j}\left(z_{2}\right) d z_{2}$ in each $\Gamma_{j}$ and let us abbreviate $H_{j}(\xi)$ in $z_{j}$. Then $z_{j, 2}$ is the only pole of $\Lambda_{\ell}$ in $\Gamma_{j}$. It is a simple one and the residue of $\Lambda_{\ell}$ at it is

$$
g_{j, \ell}=\frac{z_{j, \ell} A_{j}\left(z_{j, 2}\right)}{\xi_{1} \varphi_{j}^{\prime}\left(z_{j, 2}\right)+1}=z_{j, \ell} g_{j, 0}
$$

where $z_{j, \ell}=1$ if $\ell=0$. As $\Gamma_{j}$ is also parametrized by $H_{j}$, we can set

$$
g_{j}=\sum_{0 \leqslant \ell \leqslant 2} g_{j, \ell} d \eta_{\ell} \text { on } \Gamma_{j}
$$

and get that $g_{j, 1} / g_{j, 0}=z_{j, 1}=h_{j}$ satisfy (1.5) ; $g_{j, 2} / g_{j, 0}=z_{j, 2}=h_{j, 2}$ satisfy then $h_{j} \frac{\partial h_{j, 2}}{\partial \xi_{0}}=\frac{\partial h_{j, 2}}{\partial \xi_{1}}$ because $h_{j, 2}=-\xi_{0}-\xi_{1} h_{j}$. Note that the dependence in $\xi$ of $g$ can be made more clear if the identity $h_{j}-\varphi_{j}\left(-\xi_{0}-\xi_{1} h_{j}\right)=0$ is used. Indeed, this relation implies

$$
\left(1+\xi_{1} \varphi_{j}^{\prime}\right) \partial_{\xi_{0}} h_{j}+\varphi_{j}^{\prime}=0 \&\left(1+\xi_{1} \varphi_{j}^{\prime}\right) \partial_{\xi_{1}} h_{j}+h_{j} \varphi_{j}^{\prime}=0
$$

Hence $\varphi_{j}^{\prime}=-\left(\partial_{\xi_{0}} h_{j}\right) /\left(1+\xi_{1} \partial_{\xi_{0}} h_{j}\right)$ and

$$
\frac{1}{1+\xi_{1} \varphi_{j}^{\prime}}=\frac{\partial_{\xi_{1}} h_{j}}{\partial_{\xi_{0}} h_{j}} \frac{1+\xi_{1} \partial_{\xi_{0}} h_{j}}{h_{j}}=1+\xi_{1} \partial_{\xi_{0}} h_{j}=\partial_{\xi_{0}} h_{j, 2}
$$

since $h_{j}$ satisfies $h_{j} \partial_{\xi_{0}} h_{j}=\partial_{\xi_{1}} h_{j}$. So we have now instead of (5.3)

$$
g_{j, \ell}=A_{j}\left(H_{j}\right) h_{j, \ell} \frac{\partial h_{j, 2}}{\partial \xi_{0}}, 1 \leqslant j \leqslant p, 0 \leqslant \ell \leqslant 2 .
$$

where $h_{j, 0}=1$ for convenience.

From the definition we get that when expressed in the affine coordinates $\xi, g_{j}$ is given by the integral formula

$$
2 \pi i g_{j}=\left(\int_{\partial \Gamma_{j}} \frac{\Theta_{0}}{\xi_{0}+\xi_{1} z_{1}+z_{2}}\right) d \xi_{0}+\left(\int_{\partial \Gamma_{j}} \frac{z_{1} \Theta_{0}}{\xi_{0}+\xi_{1} z_{1}+z_{2}}\right) d \xi_{1}
$$

from which it is clear that $g_{j}$ is closed.

To achieve the proof of part A, it is enough to remark that (a2) is a direct consequence of the fact $\operatorname{Re} \Theta_{\ell}=d \widetilde{u}_{\ell}$ is exact.

5.5. Proof of B.theorem 3c. Assume that the hypothesis of (b1) is true and $\gamma$ is connected.

Case $\left.\widetilde{G}\right|_{W_{\eta_{*}}} \neq 0$. This mean $\mathcal{H} \neq \varnothing$ when $\mathcal{H}$ is minimal in the sense that no proper subset of $\mathcal{H}$ gives a decomposition of $\widetilde{G}$ with the same properties. Let $\Gamma$ be the union of the graphs $\Gamma_{j}$ of the functions $H_{j}=\left(1: h_{j}: h_{j, 2}\right)$, $1 \leqslant j \leqslant p$, where $h_{j, 2}=-\xi_{0}-\xi_{1} h_{j}$. If needed, we can choose another $\xi_{*}$ in order that $\Gamma$ does not meet $\Lambda_{\xi_{*}}$ in $\left\{w_{0}=0\right\}$. Then for any $\xi$ in a neighborhood $\Omega$ of $\xi_{*}$, the $H_{j}(\xi)$ are mutually distinct and are the points 
of $\Gamma \cap L_{\xi}$. Finally, we assume, which it is not a restriction, that $\Gamma$ has a smooth oriented boundary $\partial \Gamma$.

Let $\widetilde{\gamma}$ be the union of $\partial \Gamma$ with opposite orientation and $\gamma$ and let $\varphi_{\eta}$ be the linear function $z \mapsto \eta_{0}+\eta_{1} z_{1}+\eta_{2} z_{2}$. From the hypothesis we get directly

$$
\frac{1}{2 \pi i} \int_{\gamma} \varphi_{\eta}^{-1} \theta_{\ell}=\sum_{1 \leqslant j \leqslant p} g_{j, \ell}=\sum_{1 \leqslant j \leqslant p} g_{j, \ell} h_{j, \ell}
$$

where $h_{j, \ell}=1$ if $\ell=0$. On the other hand, if we set

$$
\Theta_{0}=\left(\partial_{\xi_{0}} h_{j, 2}\right)^{-1} g_{j, 0} d h_{j, 2}, \text { on } \overline{\Gamma_{j}}, 1 \leqslant j \leqslant p
$$

and $z_{0}=1$, the residues calculus made in the proof of part $\mathrm{A}$ implies that

$$
\int_{\partial \Gamma} z_{\ell} \varphi_{\eta}^{-1} \theta_{0}=\sum_{1 \leqslant j \leqslant p} g_{j, 0} h_{j, \ell}, 1 \leqslant j \leqslant p .
$$

Hence, $\int_{\widetilde{\gamma}} \varphi_{\eta} \theta_{\ell}=0$. As $\widetilde{\gamma}$ is contained in the affine space $E_{\xi_{*}}=\mathbb{C P}_{2} \backslash \Lambda_{\xi_{*}}$, we can apply [20, cor. 4.2 p. 265] and [11, prop. 1] and get in $E_{\xi_{*}} \backslash \widetilde{\gamma}$ a complex curve $\widetilde{\mathcal{X}}$ of finite volume where $\theta_{0}$ extends weakly in a weakly holomorphic form $\Theta_{0}$ satisfying(14)

$$
\int_{\mathcal{X}}(\bar{\partial} \varphi) \wedge \Theta_{0}=\int_{\mathcal{X}} d\left(\varphi \Theta_{0}\right)=\int_{\gamma} \varphi \theta_{0}
$$

holds for any $\varphi$ smooth in a neighborhood of $\overline{\mathcal{X}}$ and analytic near $\sigma$.

By construction, $\mathcal{X}=\widetilde{\mathcal{X}} \cup \bar{\Gamma}$ is a complex curve of $\mathbb{C P}_{2} \backslash \gamma$ where $\theta_{0}$ extends as a weakly holomorphic form $\Theta_{0}$, this extension coinciding in $\Gamma$ with the form defined by (5.5). If $\ell=1,2$, the form $\Theta_{\ell}=z_{\ell} \Theta_{0}$ is a weakly holomorphic extension of $\theta_{\ell}$ to $\mathcal{X}$.

Let $\varepsilon>0$ and let $\mathcal{W}_{\varepsilon}$ an $\varepsilon$-neighborhood of $\Lambda_{\xi_{*}}$. As $\mathcal{X}_{\varepsilon}=\mathcal{X} \backslash \overline{\mathcal{W}_{\varepsilon}}$ lies in the affine space $E_{\xi_{*}} \backslash \mathcal{W}_{\varepsilon}$, [18, th. 4.7] implies that

$$
d\left[\mathcal{X}_{\varepsilon}\right]=n_{\varepsilon}[\gamma]+\sum_{1 \leqslant j \leqslant p} n_{\varepsilon, j}\left[\gamma_{\varepsilon, j}\right]
$$

where for $1 \leqslant j \leqslant p, n_{\varepsilon}, n_{\varepsilon, j} \in \mathbb{Z}$ and $\gamma_{\varepsilon, j}$ is the (smooth) boundary of the smooth (manifold) $\mathcal{W}_{\varepsilon} \cap \Gamma_{j}$. If $0<\varepsilon^{\prime}<\varepsilon$,

$$
\begin{aligned}
\sum_{1 \leqslant j \leqslant p} d\left[\Gamma_{j} \cap\left(\mathcal{W}_{\varepsilon} \backslash \mathcal{W}_{\varepsilon^{\prime}}\right)\right] & =-d\left[\mathcal{X}_{\varepsilon}\right]+d\left[\mathcal{X}_{\varepsilon^{\prime}}\right] \\
& =\left(-n_{\varepsilon}+n_{\varepsilon^{\prime}}\right)[\gamma]+\sum_{1 \leqslant j \leqslant p}\left(-n_{\varepsilon, j}+n_{\varepsilon^{\prime}, j}\right)\left[\gamma_{\varepsilon, j}\right]
\end{aligned}
$$

Hence, $n_{\varepsilon}=n_{\varepsilon^{\prime}} \stackrel{\text { def }}{=} n$ and as each $\Gamma_{j} \cap \mathcal{W}_{\varepsilon} \backslash \mathcal{W}_{\varepsilon^{\prime}}$ is a smooth manifold with boundary, $n_{\varepsilon, j}=n_{\varepsilon^{\prime}, j}=-1$. Taking now limits in (5.7) when $\varepsilon$ goes to zero, we get $d[\mathcal{X}]=n[\gamma]$. We suppress from $\mathcal{X}$ any compact component it may have and still denote the result by $\mathcal{X}$; note that $\mathcal{X}$ has now to be

\footnotetext{
${ }^{14} \operatorname{In}[20][1], 5.6$ is in fact obtained only for $\varphi$ smooth on $\mathcal{X}$ and holomorphic in a neighborhood of $\gamma$ but (5.6) follows from this together with (5.4) and the residue relations (very close in spirit to the relations $T_{m, \xi}$ )

$$
\frac{1}{2 \pi i} \int_{\gamma} z_{1}^{m} \frac{\theta_{0}}{\xi_{0}+\xi_{1} z_{1}+z_{2}}=\sum_{1 \leqslant j \leqslant p} h_{j}^{m}(\xi) g_{j, 0}(\xi)
$$

where $h_{j}(\xi), h_{j, 2}(\xi)$ and $g_{j, 0}(\xi)$ are as above ; indeed these relations enable for generic $\xi$ the computation of $g_{j, 0}(\xi)$ by a kramerian system and hence imply the smoothness of $\Theta_{0}$ near points of $\gamma \backslash \sigma$. However, this precision is used not essentialy in the sequel.
} 
connected. Since $\mathcal{X}$ is a complex curve of $\mathbb{C P}_{2} \backslash \gamma, 10$ implies that if $n \neq 0$, there is in $\gamma$ a compact set $\sigma$ such that $h^{1}(\sigma)=0$ and $(\overline{\mathcal{X}}, \pm \gamma)$ is manifold with boundary near points of $\gamma \backslash \sigma$; as $\mathcal{X}$ is connected, this implies $n= \pm 1$. When $n=0$, the structure theorems of Harvey-Shiffman [17] implies that $\mathcal{Z}=\overline{\mathcal{X}}$ is then a complex compact curve of $\mathbb{C P}_{2}$; since $\gamma$ is smooth, $\gamma$ is locally a Jordan curve of $\mathcal{Z}_{\text {reg }}$ and the points where $\gamma$ may meet the finite set $\mathcal{Z}_{\text {sing }}$ are only self-intersection points of $\mathcal{Z}$.

Since $\mathcal{X} \cap \Lambda_{\xi}=\left\{\left(1: h_{j}(\xi): h_{j, 2}(\xi)\right), 1 \leqslant j \leqslant p\right\}$, the Stokes's formula gives $G=\Sigma h_{j}$ (see proof of theorem 3 a.A).

Assume that $\left(\partial^{2} G / \partial \xi_{0}^{2}\right)_{\mid W_{\xi_{*}}} \neq 0$. Then $n \neq 0$ because otherwise for $\xi$ closed to $\xi_{*}$, the intersection of $\mathcal{X}$ with the line $\Lambda_{\xi}$ should have to be the intersection with $\Lambda_{\xi}$ of a compact Riemann surface, namely $\overline{\mathcal{X}}$, which by a theorem of Reiss would force $\Sigma h_{j}$ to be affine in $\xi_{0}$ (see [16, ch. 5.2] or [20, section 2]). Reasoning like in the proof of 3a.B, we also eliminate the possibility $d[\mathcal{X}]=-[\gamma]$ because it would imply that if $\mathcal{H}=\left\{h_{1}, \ldots, h_{p}\right\}$ is minimal in the sense that no proper subset of $\mathcal{H}$ satisfy (a1), each $h_{j}$ also satisfies $h_{j, y}=-h_{j, x} h_{j}$. Hence $n=1$.

When $\left(\partial^{2} G / \partial \xi_{0}^{2}\right)_{\mid W_{\xi_{*}}}=0$, that is when $\Sigma h_{j}$ is affine in $\xi_{0}$, and when $\overline{\mathcal{X}}$ is not an algebraic curve where $\gamma$ is a slit, $n$ has to be non null and hence is \pm 1 . Since $\left.\widetilde{G}\right|_{W_{\eta_{*}}} \neq 0$, the minimal $\mathcal{H}$ in the above sense is not empty and reasoning likewise, we get $d[\mathcal{X}]=[\gamma]$.

Case $\left.\widetilde{G}\right|_{W_{\eta_{*}}}=0$. This means that the minimal $\mathcal{H}$ is empty. Then, we can apply [20, th. 4.2 p. 264] in the affine case (see [11] for a generalization and a detailed proof in this case) to get in $\mathbb{C}^{2} \backslash \gamma$ (here $\mathbb{C}^{2}$ is the complement of $\Lambda_{\xi_{*}}=\left\{\xi_{* 0} w_{0}+\xi_{1 *} w_{1}+w_{2}=0\right\}$ in $\left.\mathbb{C P}_{2}\right)$ a complex curve $\mathcal{X}$ of finite volume where $\theta_{0}$ extends weakly in a weakly holomorphic form $\Theta_{0}$ satisfying (5.6).

Since $\theta_{0}$ yields a non zero measure on $\gamma$ which, because $\left.\widetilde{G}\right|_{W_{\eta_{*}}}=0$, is orthogonal to all polynomials of $\mathbb{C}^{2} \sim \mathbb{C P}_{2} \backslash \Lambda_{\xi_{*}}$, we can apply Bishop [7] and [26] (Wermer originates and solves this problem [29] for the real analytic case) to get that $\mathcal{X}$ is the polynomial hull $\widetilde{\gamma}$ of $\gamma$ in $\mathbb{C}^{2}$ and $d( \pm[\mathcal{X}])=[\gamma]$. [18] (see also [10]) implies then that $\gamma$ contains a compact set $\sigma$ such that $h^{1}(\sigma)=0$ and $(\overline{\mathcal{X}}, \gamma)$ is manifold with boundary near points of $\gamma \backslash \sigma$.

To prove (b2), we go back to the assumption that (a1) is true and we assume in addition that $\Theta_{\ell}$ is holomorphic and satisfies (1.8) for all $c \in$ $H_{1}\left(\mathcal{X}_{\text {reg }}\right)$. Then, there exists $U_{\ell} \in C^{\infty}\left(\mathcal{X}_{\text {reg }}\right)$ such that $\Theta_{\ell}=d V_{\ell}$ on $\mathcal{X}_{\text {reg }}$; since $\Theta_{\ell}$ is a $(1,0)$-form, $\partial V_{\ell}=d V_{\ell}=\Theta_{\ell}$. We know from the preceding point that $d[\mathcal{X}]=n[\gamma]$ where $n \in\{0,1\}$, up to a change of orientation of $\gamma$ when $\left.\widetilde{G}\right|_{W_{\eta_{*}}}=0$. Assume at first that $n=1$. As $d$ is elliptic up to the boundary, $V_{\ell}$ has to be smooth up to the boundary in the classical sense near points of $\gamma$ outside $\sigma$ and the preceding equality (5.6) yields $d v_{\ell}=d u_{\ell}$ where $v_{\ell}=\left.V_{\ell}\right|_{\gamma}$. Hence, $z_{0}$ is a point of $\gamma$ where $(\overline{\mathcal{X}}, \gamma)$ is a manifold with boundary, there is a constant $c$ such that $v_{\ell}=u_{\ell}+c$ near $z_{0}$ in $\gamma$. Since $h^{1}(\sigma)=0$ and $d v_{\ell}=d u_{\ell}$ is smooth we have $v_{\ell}(z)-v_{\ell}\left(z_{0}\right)=\int_{\gamma_{z_{0}, z}} d u_{\ell}$ where $\gamma_{z_{0}, z}$ is the positively oriented path of $\gamma$ starting at $z_{0}$ and ending at $z$. Hence, $v_{\ell}(z)-u_{\ell}\left(z_{0}\right)-c=u_{\ell}(z)-u_{\ell}\left(z_{0}\right)$ and $v_{\ell}(z)=u_{\ell}(z)+c$. This implies that $U_{\ell}$ is a weakly harmonic extension of $u_{\ell}$. When $n=0, \overline{\mathcal{X}}$ is 
two sided locally near points of $\gamma$ and each local side has the same boundary regularity as in the case $n=1$. Hence, we can reason as in this case and get that there is a weakly harmonic extension of $u_{\ell}$ to $\mathcal{X}$.

When we assume also that $\int_{\mathcal{X}_{\text {reg }}} \Theta_{\ell} \wedge \overline{\Theta_{\ell}}<+\infty, \Theta_{\ell}$ is holomorphic in the sense that its pullback to any normalization $\pi: \mathcal{Z} \rightarrow \mathcal{X}$ of $\mathcal{X}$ is holomorphic and not only meromorphic. The isolated singularities that the pullback $V_{\ell}$ of $U_{\ell}$ may have in $\mathcal{Z}$ are removable because $d V_{\ell}$ is smooth. So $U_{\ell}$ is harmonic on $\mathcal{X}$ and $U_{\ell}$ is the harmonic extension of $u_{\ell}$ to $\mathcal{X}$. The proof is complete.

5.6. Proof of theorem 3b. The map $f$ enable us to embed the abstract IDN-problem of theorem $3 \mathrm{~b}$ in the projective but concrete frame of theorem 3d. So theorem 3b.A is a direct consequence of 3c. A.

For the converse part B.b1, we apply 3 d.B to $\delta=f(\gamma)$ and $\theta_{\ell}=\theta u_{\ell}$, $\ell=0,1,2$. We get in $\mathbb{C P}_{2} \backslash \delta$ an irreducible complex curve $\mathcal{Y}$ such that $d[\mathcal{Y}]=n[\delta]$ where $n \in\{0,1\}$, up to a change of orientation when $\left.\widetilde{G}\right|_{W_{\eta_{*}}}=$ 0 ; in addition, each $\theta_{\ell}$ extends weakly to $\mathcal{Y}$ into a weakly holomorphic $(1,0)$-form $\Theta_{\ell}^{\mathcal{Y}}$.

When $n=1$, the boundary regularity of $\mathcal{Y}$ mentioned in the proof of theorem $3 \mathrm{~g}$. B enable to apply readily the construction made in the proof of theorem $3 \mathrm{a}$ : adding to $\mathcal{Y}$ a subset $\sigma^{\prime}$ of $\gamma$ of zero one dimensional Hausdorff measure, we get an abstract complex curve $\widetilde{\mathcal{Y}}$ which can be normalized in the classical sense into an abstract Riemann surface $\mathcal{X} ; \gamma$ can then be topologically glued to $\mathcal{X}$ so that $(\overline{\mathcal{X}}, \gamma)$ becomes a manifold with almost smooth boundary where the pullback $F$ to $\mathcal{X}$ of the meromorphic map $\mathbb{C P}_{2} \ni$ $z \mapsto\left(z_{1}, z_{2}\right)$ gives a meromorphic extension of $f$ to $\mathcal{X}$. Since the forms $\Theta_{\ell}^{\mathcal{Y}}$ are meromorphic on $\mathcal{Y}, \Theta_{\ell}=F^{*} \Theta_{\ell}^{\mathcal{Y}}$ is well defined and meromorphic outside $\mathcal{X} \backslash F^{-1}\left(\sigma^{\prime}\right)$ which has zero length.

When $n=0, \mathcal{Z}=\overline{\mathcal{X}}$ is an algebraic curve and one can use a standard normalization of $\mathcal{Z}$ to get the same kind of conclusions.

The supplementary hypothesis of part B.b2 force each $\Theta_{\ell}$ to have only removable singularities. Reasoning like in the proof of 3d.B.b2, (1.8) implies that $\Theta_{\ell}=d V_{\ell}=\partial V_{\ell}$ for some harmonic function $V_{\ell}$ smooth up to regular boundary points of $\overline{\mathcal{X}}$ (when $n=0, \gamma$ cuts locally $\mathcal{X}$ into two domains and this means that each restriction of $V_{\ell}$ to these domains is smooth up to $\gamma$ ) and that there is a constant $c$ such that $U_{\ell}+c$ agrees with $u_{\ell}$ on $\gamma \cdot \square$

\section{Characterizations, effective or affine}

6.1. Explicit integro-differential characterization. In this section where th. 4 is proved, $\left(\xi_{0}, \xi_{1}\right)$ is replaced by the simpler $(x, y)$ and we reason in a neighborhood of $(0,0) \cdot \mathcal{O}_{0}$ is the set of one variable holomorphic functions near 0 is denoted by, $\mathcal{O}_{0} \otimes \mathbb{C}_{d}[Z]$ stands for the set of polynomials of degree at most $d$ with independent variable $Z$ and coefficients in $\mathcal{O}_{0}$. An element of $\mathcal{O}_{0} \otimes \mathbb{C}_{d}[X]$ (resp. $\mathcal{O}_{0} \otimes \mathbb{C}_{d}[Y]$ ) should be think as a function of the type $(x, y) \mapsto \sum_{0 \leqslant j \leqslant d} \lambda_{j}(x) y^{j}$ (resp. $\left.(x, y) \mapsto \sum_{0 \leqslant j \leqslant d} \lambda_{j}(y) x^{j}\right)$ where each $\lambda_{j} \in \mathcal{O}_{0}$.

If $h$ is differentiable, the derivative of $h$ with respect to one of its variable $u$ is denoted $h_{u}$. If $U$ is an open set in $\mathbb{C}^{n}, \mathcal{O}(U)$ is the space of holomorphic functions in $U$; if $h \in \mathcal{O}(U)$ and $0 \in U$, we set $h_{x^{-0}, y^{0}}=h$ and in any simply 
connected neighborhood of 0 in $U$, we denote by $h_{x^{-\alpha-1}, y^{\beta}}(\alpha, \beta \in \mathbb{N})$ the function which vanishes at 0 and satisfies $\partial\left(h_{x^{-\alpha-1}, y^{\beta}}\right) / \partial x=h_{x^{-\alpha}, y^{\beta}}$; $h_{x, \alpha y^{-\beta-1}}$ is defined similarly.

A two variable function $h$ is called a shock wave function on a domain $D$ of $\mathbb{C}^{2}$ if it is holomorphic and satisfies $h_{y}=h_{x} h$ on $D$.

A $p$-algebrö̈de function on $D$ is a $p$-uple $h=\left(h_{1}, \ldots, h_{m}\right)$ of functions from $D$ to $\mathbb{C}$ for which one can find $p$ holomorphic functions $a_{0}, \ldots, a_{p-1}$ in $D$ such that for $z \in D, h_{1}(z), \ldots, h_{p}(z)$ are the roots, with multiplicities, of the polynomial $T^{h}=X^{p}+a_{p-1}(z) X^{p-1}+\cdots+a_{1}(z) X+a_{0}(z)$.

A p-multivaluate shock wave function on $D$ is a $p$-algebroïde function $h$ on $D$ such that $\Sigma=\left\{\operatorname{Discr} T^{h}=0\right\}$ is a hypersurface of $D$ and for any $z_{*} \in D \backslash \Sigma$, the holomorphic functions $h_{1}^{*}, . ., h_{p}^{*}$ which near $z_{*}$ describe the roots of $T_{z}^{h}$ are non null shock wave functions; the first symmetric function of $T^{h}$, that is the sum of the roots of $T^{h}$, is called the trace of $T^{h}$ of $h$. Traces of $p$-multivaluate shock wave functions are called $p$-shock wave functions.

Lemma 16. Consider $p$ mutually distinct functions $h_{1}, \ldots, h_{p}$ holomorphic in a domain $D$ of $\mathbb{C}^{2}$. Then, each $h_{j}$ is a shock wave function if and only the functions $\sigma_{k}=(-1)^{k} \sum_{1 \leqslant j_{1}<\cdots<j_{k} \leqslant p} h_{j_{1}} \ldots h_{j_{k}}$ satisfy the following system

$$
\sigma_{p} \sigma_{1, x}+\sigma_{p, y}=0 \quad \& \quad \sigma_{k} \sigma_{1, x}+\sigma_{k, y}=\sigma_{k+1, x}, 1 \leqslant k \leqslant p-1 .
$$

Proof. Set $T=X^{p}+\sum_{1 \leqslant k \leqslant p} \sigma_{k} X^{p-k}$ and $T^{\prime}=\partial T / \partial X$. The relations $0=$ $(T h)_{x}=(T h)_{y}$ and $h^{p}=-\sum_{1 \leqslant k \leqslant p} \sigma_{k} h^{p-k}$ yields $\left(T^{\prime} h\right)\left(h_{y}-h h_{x}\right)=S h$ with

$$
S=\sum_{1 \leqslant k \leqslant p-1}\left[\sigma_{k+1, x}-\sigma_{k} \sigma_{1, x}-\sigma_{k, y}\right] X^{p-k}-\left(\sigma_{p} \sigma_{1, x}+\sigma_{p, y}\right)
$$

Since $\operatorname{deg} S \leqslant p-1$, the fact that each $h_{j}$ is a shock wave function implies that the coefficients of $S$ vanish in a non empty open set and thus in the domain $D$. If $S=0$ at every point of $D$, then each $h_{j}$ verifies $h_{y}-h_{x} h=0$ in the domain $D$ because $T^{\prime} h_{j} \not \equiv 0$.

Proposition 17. Let $D$ be a simply connected domain of $\mathbb{C}^{2}$ containing 0 , $\Delta$ its image by the projection $(x, y) \mapsto y$ and and $H \in \mathcal{O}(D)$. When $u$ is differentiable, we set

$$
\mathcal{D}_{H} u=e^{H_{x, y^{-1}}} \partial\left(u e^{-H_{x, y^{-1}}}\right) / \partial y \quad \& \quad \mathcal{L}_{H} u=\left(\mathcal{D}_{H} u\right)_{x^{-1}}
$$

The following two assertions are equivalent

1. $H$ is a p-shock wave function in $D$.

2. There exists $\lambda_{1}, \ldots, \lambda_{p-1} \in \mathcal{O}(\Delta)$ such that for $\widetilde{\lambda}_{j}(x, y)=\lambda_{j}(y)$, $1 \leqslant j \leqslant p-1$,

$$
\begin{gathered}
\mathcal{D}_{H} \mathcal{L}_{H}^{p-1} H=\mathcal{D}_{H} \mathcal{L}_{H}^{p-2} \widetilde{\lambda}_{1}+\cdots+\mathcal{D}_{H} \mathcal{L}_{H}^{0} \widetilde{\lambda}_{p-1} \\
\operatorname{Discr} T_{z} \not \equiv 0
\end{gathered}
$$

where $T_{z}=X^{p}+\sum_{1 \leqslant k \leqslant p} s_{k}(z) X^{p-k}$ with

$$
s_{k}=-\mathcal{L}_{H}^{k-1} H+\mathcal{L}_{H}^{k-2} \widetilde{\lambda}_{1}+\cdots+\mathcal{L}_{H}^{0} \widetilde{\lambda}_{k-1}, 1 \leqslant k \leqslant p .
$$

More precisely, in case (2) is true, $T$ determines a p-multivaluate shock wave function with trace $H$. Conversely, if $H$ is the trace of a $p$-multivaluate 
shock wave function $T$, the $p$ holomorphic functions which near a point $z_{*}$ in $\{$ Discr $T \neq 0\}$ describes the roots of $T_{z}$ have symmetric functions $(-1)^{k} s_{k}, 1 \leqslant$ $k \leqslant p$ which satisfy (6.2) and (6.4).

Proof. 1) Assume $H$ is a $p$-shock wave function in $D$. Then, $H$ is the first symmetric function of some $T \in \mathcal{O}(D) \otimes \mathbb{C}_{p}[Z], \Sigma=\{\operatorname{Discr} T=0\} \neq D$ and for any fixed $z_{*} \in D \backslash \Sigma, \operatorname{deg} T_{z_{*}}=p$ and the holomorphic functions $h_{1}^{*}, . ., h_{p}^{*}$ which near $z_{*}$ describe the roots of $T_{z}$ are non null shock wave functions with no common value on a sufficient small convex neighborhood $W=U \times V$ of $z_{*}$. For $k \in\{1, \ldots, p\}$ and on $W$, set $\rho_{k}=\sigma_{k} e^{-H_{x, y^{-1}}}$ where $\sigma_{k}$ is defined in lemma 16. Then (6.1) implies that $\rho_{p, y}=0$ and that for $k \in\{1, \ldots, p-1\}$,

$$
\left(\rho_{k+1} e^{H_{x, y^{-1}}}\right)_{x}=-H_{x} \sigma_{k}+\sigma_{k, y}=e^{H_{x, y^{-1}}} \frac{\partial}{\partial y} \sigma_{k} e^{-H_{x, y^{-1}}}=e^{H_{x, y^{-1}} \rho_{k, y}}
$$

which yields $\lambda_{k} \in \mathcal{O}(V)$ such that

$$
\rho_{k+1}(x, y) e^{H_{x, y^{-1}}}=\left[e^{H_{x, y^{-1}}} \rho_{k, y}\right]_{x^{-1}}+\lambda_{k}(y) .
$$

Since $\sigma_{1}=-H$, we get $e^{-H_{x, y^{-1}}}\left[e^{H_{x, y^{-1}}} \rho_{1, y}\right]_{x^{-1}}=-e^{-H_{x, y^{-1}}} \mathcal{L}_{H} H$. Setting $\widetilde{\lambda}_{0}=-H$, we obtain $\rho_{2}=e^{-H_{x, y^{-1}}}\left(\mathcal{L}_{H} \lambda_{0}+\widetilde{\lambda}_{1}\right)$ and a straightforward finite recurrence gives (6.4) with $\left(s_{k}\right)=\left(\sigma_{k}\right)$. In particular, $k=p$ yields (6.2), because $\rho_{p, y}=0$; the discriminant of $T_{z}$ don't vanish in $W$ because $h_{1}, \ldots, h_{p}$ have no common value. Since (6.4) also reads $\sigma_{k+1}=\mathcal{L}_{H} \sigma_{k}+\widetilde{\lambda}_{k}$ we obtain that $\lambda_{k}=\sigma_{k+1}(0,$.$) on V$. Hence, $\lambda_{1}, \ldots, \lambda_{p}$ do not depend on $z_{*}$ so that they are well defined holomorphic function on $\Delta$.

2) Assume now (2) is true. We only have to check that $T=X^{p}-$ $\sum_{1 \leqslant k \leqslant p} s_{k} X^{p-k}$ is actually a $p$-multivaluate shock wave function. Formulas (6.4) also read $s_{k+1}=\mathcal{L}_{H} s_{k}+\widetilde{\lambda}_{k}$ for $1 \leqslant k \leqslant p-1$ and (6.2) means that $\mathcal{D}_{H} s_{p}=0$. Hence $0=s_{p, y}-H_{x} s_{p}=s_{p, y}-s_{1, x} s_{p}$ and $s_{k+1, x}=\mathcal{D}_{H} s_{k}=$ $s_{k, y}-H_{x} s_{k}=s_{k, y}+s_{1, x} s_{k}$. So, if $z_{*} \in D$ is outside $\Sigma=\{\operatorname{Discr} T=0\}$, lemma 16 implies that the holomorphic functions $h_{1}, \ldots, h_{p}$ which near $z_{*}$ describe the roots of $T_{z}$ are mutually distinct shock wave function.

The following describes $p$-shock wave functions which are affine in $x$.

Proposition 18. Let $D$ be a simply connected domain of $\mathbb{C}^{2}$ containing 0 , $a, b \in \mathcal{O}(D)$ and $H=x \otimes a+1 \otimes b$. Then, $H$ is a p-shock wave function, if and only if there exists $Q_{0}, Q_{1} \in \mathbb{C}_{p-1}[Y]$ such that

$$
\begin{aligned}
a= & \frac{Q_{1}}{1-Q_{1, y^{-1}}} \& b=\frac{Q_{0}}{1-Q_{1, y^{-1}}} \\
& \operatorname{Discr}\left(1-Q_{1, y^{-1}}\right) \neq 0,
\end{aligned}
$$

In addition, when (6.5) and (6.6) are satisfied, the decomposition of $H$ in elementary fractions gives $H$ as a sum of shock wave functions.

Proof. Assume $H=\sum_{1 \leqslant j \leqslant p} h_{j}$ where $h_{1}, \ldots, h_{p}$ are mutually distinct shock wave functions ; with proposition 17 notations, $(-1)^{1} s_{1}, \ldots,(-1)^{p} s_{p}$ are the symmetric functions of $h_{1}, \ldots, h_{p}$ and satisfy the relations $\mathcal{D}_{H} s_{p}=0$ and $s_{k+1}=\mathcal{L}_{H} s_{k}+\widetilde{\lambda}_{k}, 1 \leqslant k \leqslant p-1$. There exist sequences of holomorphic 
functions, $\left(\lambda_{j, k}\right)_{k \in \mathbb{N}},\left(a_{k}\right)_{k \in \mathbb{N}}$ and $\left(b_{k}\right)_{k \in \mathbb{N}}$, each satisfying the recurrence $u_{k+1}=u_{k}^{\prime}-a u_{k}$, such that

$$
\mathcal{L}_{H}^{k} \widetilde{\lambda}_{j}=\frac{x^{k}}{k !} \otimes \lambda_{j, k}, \quad \mathcal{L}_{H}^{k} H=\frac{x^{k+1}}{(k+1) !} \otimes a_{k}+\frac{x^{k}}{k !} \otimes b_{k}, k \in \mathbb{N}
$$

Hence, (6.2) yields the vanishing of the $x$-polynomial

$$
\frac{x^{p}}{p !} \otimes a_{p}+\frac{x^{p-1}}{(p-1) !} \otimes b_{p}-\sum_{1 \leqslant j \leqslant p-1} \frac{x^{p-1-j}}{(p-1-j) !} \otimes \lambda_{j, p-j}
$$

and ensures $a_{p}=b_{p}=\lambda_{j, p-j}, 1 \leqslant j \leqslant p-j$. So, one can find $Q_{1}, Q_{0} \in$ $\mathbb{C}_{p-1}[Y]$ and $\Lambda_{j} \in \mathbb{C}_{p-j-1}[Y], 1 \leqslant j \leqslant p-1$, such that with $A=a_{y^{-1}}$

$$
a_{k}=Q_{1}^{(k)} e^{A}, b_{k}=Q_{0}^{(k)} e^{A}, \lambda_{j, k}=\Lambda_{j}^{(k)} e^{A}, k \in \mathbb{N} .
$$

Thus $a=a_{0}=Q_{1} e^{A}, 1-e^{-A}=Q_{1, y^{-1}}$ and hence, (6.5).

Conversely, assume that (6.5) and (6.6) are true for some $Q_{0}, Q_{1} \in \mathbb{C}_{p-1}[Y]$. Then, the decomposition in elementary fraction of $H$ is $\Sigma h_{j}$ where $h_{j}(x, y)=$ $\frac{q_{j} x+c_{j}}{1-q_{j} y}, 1 \leqslant j \leqslant p$ and $q_{1}^{-1}, \ldots, q_{p}^{-1}$ are the roots of $1-Q_{1, y^{-1}}$. It is quite evident that $h_{1}, \ldots, h_{p}$ are mutually distinct shock wave functions.

Remark 1. If $x \otimes a+1 \otimes b$ is the sum of $p$-mutually distinct shock wave functions $h_{1}, \ldots, h_{p}$, each $h_{j}$ is algebraic since for $1 \leqslant k \leqslant p$, 6.4 yields $s_{k}(x, y)=\left[1-Q_{1 y^{-1}}(y)\right]^{-1} S_{k}(x, y)$ where $S_{k}$ is the polynomial defined by

$$
S_{k}=-\frac{x^{k} \otimes Q_{1}^{(k-1)}}{k !}-\frac{x^{k-1} \otimes Q_{0}^{(k-1)}}{(k-1) !}+\sum_{1 \leqslant j \leqslant k-1} \frac{x^{k-1-j} \otimes \Lambda_{j}^{(k-1-j)}}{(k-1-j) !} .
$$

Remark 2. When $Q_{1}$ is allowed to be non generic, $1-Q_{1, y^{-1}}$ can only be written in the form $1-Q_{1, y^{-1}}=\prod_{1 \leqslant j \leqslant m}\left(1-q_{j} y\right)^{\alpha_{j}}$ with $\alpha_{1}, \ldots, \alpha_{p} \in \mathbb{N}^{*}$. Hence, if $Q_{0} \in \mathbb{C}_{p-1}[Y]$ and $(a, b)$ is defined by (6.5), there is constants $c_{j, \ell}$ such that

$$
H \stackrel{\text { def }}{=} x \otimes a+1 \otimes b=\sum_{1 \leqslant j \leqslant m} \sum_{1 \leqslant \ell \leqslant \alpha_{j}} h_{j, \ell}
$$

where $h_{j, \ell}=\frac{q_{j} x}{1-q_{j} y}+\frac{c_{j, \ell}}{\left(1-q_{j} y\right)^{\ell}}, 1 \leqslant j \leqslant m, 1 \leqslant \ell \leqslant \alpha_{j}$. Each $h_{j, \ell}$ is now a generalized shock wave function in the sense it is a solution of the equation

$$
h_{y}-h_{x} h=(\ell-1) \kappa h_{x}^{\ell+1}
$$

where $\kappa \in \mathbb{C}$ is equal to $c_{j, \ell} / q_{j}^{\ell}$.

Generalized shock wave functions arise when an affine function $H=x \otimes$ $a+1 \otimes b$ has coefficient $a$ and $b$ given by (6.5) not constraint to (6.6). In that case, $H$ is clearly a limit of $p$-shock wave functions. The lemma below, which is an elementary consequence of prop. 18, proves the converse and so, shows that generalized shock wave functions occur naturally.

Lemma 19. Let $\left(H^{t}\right)_{t \in T}=\left(x \otimes a^{t}+1 \otimes b^{t}\right)_{t \in T}$ be a continuous family of holomorphic affine functions in a simply connected domain D such that the set $T_{\text {reg }}$ of parameters $t$ for which $H_{t}$ is a p-shock wave function is dense in $T$, then there exists in $\mathbb{C}_{p-1}[Y]$ continuous family of holomorphic polynomials $\left(Q_{1}^{t}\right)$ and $\left(Q_{0}^{t}\right)$ such that for any $t \in T, a^{t}=Q_{1}^{t}\left(1-Q_{1, y^{-1}}^{t}\right)^{-1}$ and $b^{t}=$ 
$Q_{1,0}^{t}\left(1-Q_{1, y^{-1}}^{t}\right)^{-1}$. Hence, $H^{t}$ is a p-shock (resp. p-generalized) shock wave function when Discr $\left(1-Q_{1, y^{-1}}^{t}\right) \neq 0$ (resp. Discr $\left(1-Q_{1, y^{-1}}\right)=0$ ).

6.1.1. Proof of theorem 4 . 1) Assume that there is an open Riemann surface $\mathcal{X}$ such that $\overline{\mathcal{X}}=\mathcal{X} \cup \gamma$ is a manifold with almost smooth boundary. Then, theorem 3 a gives that almost all point $\left(\xi_{0 *}, \xi_{1 *}, 1\right)$ of $\mathbb{C P}_{2}$ has a neighborhood $W_{\xi_{*}}$ for which one can find an integer $p$ and $p$ mutually distinct shock wave functions $h_{1}, \ldots, h_{p}$ on $W_{\xi_{*}}$ such that $L=\Sigma h_{j}-G$ is affine in $\xi_{0}$. Set $s_{k}=(-1)^{k} \sum_{1 \leqslant j_{1}<\cdots<j_{k} \leqslant p} h_{j_{1}} \ldots h_{j_{k}}$ and $T=X^{p}+\sum_{1 \leqslant k \leqslant p} s_{k} X^{p-k}$. Then $\operatorname{Discr} T \not \equiv 0$ and proposition 17 implies that property (1) of th. 6 holds.

Assume now that $\widetilde{p}$ is the least integer $q$ such that there is a $q$-multivaluate shock wave function whose trace differs from $\left.G\right|_{W_{*}}$ only by a $\xi_{0}$-affine function. Let $\widetilde{T}$ be a $\widetilde{p}$-multivaluate shock wave function with trace $\widetilde{H}$ such that $\widetilde{L}=\widetilde{H}-\left.G\right|_{W_{\xi_{*}}}$ is affine in $\xi_{0}$. Let $\widetilde{h}_{1}, \ldots, \widetilde{h}_{\widetilde{p}}$ be the holomorphic function on $W_{\xi_{*}}$ which describes the roots of $\widetilde{T}$. Then $\left\{\widetilde{h}_{1}, \ldots, \widetilde{h}_{\widetilde{p}}\right\}$ is minimal in the sense of the fourth remark below theorem 3 a.

When $\widetilde{p} \geqslant 1$, this remark says that $\mathcal{X}$ is a normalization of the analytic extension $\mathcal{Y}$ in $\mathbb{C P}_{2} \backslash f(\gamma)$ of the union of the graphs of the functions $\left(1: \widetilde{h}_{j}:-\xi_{0}-\xi_{1} \widetilde{h}_{j}\right), 1 \leqslant j \leqslant \widetilde{p}$, and for any $\xi \in W_{\xi_{*}}$, the intersection of $\mathcal{Y}$ with the projective lines $\xi_{0} w_{0}+\xi_{1} w_{1}+w_{2}=0$ is

$$
\left\{\left(1: \widetilde{h}_{j}(\xi): \xi_{0}-\xi_{1} \widetilde{h}_{j}(\xi)\right) ; 1 \leqslant j \leqslant \widetilde{p}\right\} .
$$

Since by prop $10 \mathcal{Y}$ is uniquely determined by $(\gamma, f)$, each $\widetilde{h}_{j}$ and so, each symmetric function $\widetilde{s}_{k}$ of $\widetilde{h}_{1}, \ldots, \widetilde{h}_{k}$, is uniquely determined by $(\gamma, f)$. If $\lambda_{1}, \ldots, \lambda_{\widetilde{p}}$ are any one variable holomorphic function such that assertion (2) of prop. 17 holds, with $\widetilde{H}$ instead of $H$, then $\lambda_{k-1}\left(\xi_{1}\right)=\widetilde{s}_{k}\left(0, \xi_{1}\right), 1 \leqslant k \leqslant$ $p-1$. Hence, $\lambda_{1}, \ldots, \lambda_{\widetilde{p}}$ are uniquely determined by $(\gamma, f)$.

Lemma 13 implies now that $L$ is affine in $\xi_{0}$ and is, for some integer $q$, the limit of a continuous one parameter family of $\xi_{0}$-affine $q$-shock wave functions. Thanks to lemma 19, this implies that $L\left(\xi_{0}, \xi_{1}\right)=\xi_{0} \frac{\alpha^{\prime}\left(\xi_{1}\right)}{1-\alpha\left(\xi_{1}\right)}+$ $\frac{\beta\left(\xi_{1}\right)}{1-\alpha\left(\xi_{1}\right)}$ where $\alpha \in \mathbb{C}_{q}\left[\xi_{1}\right]$ vanish at zero and $b \in \mathbb{C}_{q-1}\left[\xi_{1}\right]$.

2) Assume now that property (1) of th. 国holds. Set $T=X^{p}+\sum_{1 \leqslant k \leqslant p} s_{k} X^{p-k}$ where now $s_{k}$ are defined by (6.4). Proposition 17 implies then that $T$ is determines a $p$-multivaluate shock wave function whose trace is $G+L$. Since $L$ is affine in $\xi_{0}, G$ satisfies the hypothesis of theorem 3 a.B. As $G$ is not affine in $\xi_{0}$ by hypothesis, $\gamma$, with its given orientation, is the boundary of an open Riemann surface $\mathcal{X}$ with the sought properties. Hence, (4) has to be satisfied from the direct part of theorem 4

6.1.2. Particular case of theorem 日. For minimal $p$ equal to 2 , it turns out that theorem 1 says that $\gamma$ bounds almost smoothly an open Riemann surface $\mathcal{X}$ where $f$ extends meromorphically if and only if for some constants $c, \alpha_{1}, \alpha_{2}, \beta_{0}, \beta_{1}$

$$
\mathcal{D}_{G} \mathcal{L}_{G} G=c C+\alpha_{1} A_{1}+\alpha_{2} A_{2}+\beta_{0} B_{0}+\beta_{1} B_{1}
$$


where

$$
\begin{aligned}
C & =\left(G_{x}-g_{x}\right) \mathrm{e}^{g_{x, y}-1} \\
A_{1} & =y \mathcal{D}_{G} \mathcal{L}_{G} G+\left(x \mathcal{L}_{G} G\right)_{x}, A_{2}=y^{2} \mathcal{D}_{G} \mathcal{L}_{G} G+\left(2 x y \mathcal{L}_{G} G+x^{2} G\right)_{x} \\
B_{0} & =\mathcal{D}_{G} G-\mathcal{D}_{G} g, B_{1}=y B_{0}+g-G
\end{aligned}
$$

6.2. Affine characterization. A characterization for an affine presentation is possible but very special data have to be selected. Assume $\mathcal{X}$ is a Riemann surface with almost smooth boundary $\gamma$. When $u \in C^{\infty}(\gamma)$, a straightforward computation gives that $d^{c} \widetilde{u}=(N u) \tau^{*}$ as forms of $\gamma \backslash \overline{\mathcal{X}}_{\text {sing }}$. So, lemma 11 implies that

$$
\int_{\gamma}(N u) \tau^{*}=\int_{\mathcal{X}} d d^{c} \widetilde{u}=0
$$

Hence $(N u) \tau^{*}$ has a primitive $v$ on $\gamma$ and the holomorphic extension to $\mathcal{X}$ of $h=u+i v$ is equivalent to the moments condition

$$
\forall \Psi \in H^{1,0}(\overline{\mathcal{X}}), \int_{\gamma} h \Psi=0 .
$$

If $H \in \mathcal{O}(\overline{\mathcal{X}})^{N+1}$ is such that $h=\left.H\right|_{\gamma}$ embeds $\gamma$ in $\mathbb{C}^{N+1}, \mathcal{Y}=H(\mathcal{X}) \backslash h(\gamma)$ is a complex curve of $\mathbb{C}^{N+1} \backslash h(\gamma)$ which has finite volume and has boundary $h(\gamma)$ and $\gamma$ has to satisfy the Harvey-Lawson-moments condition

$$
\forall k_{0}, \ldots, k_{N} \in \mathbb{N}, \int_{\gamma} h_{0}^{k_{0}} \ldots h_{N}^{k_{N}} d h_{0}=0
$$

which by the way contains also (6.8).

Proposition 20. Let $u_{0}, \ldots, u_{N} \in C^{\infty}(\gamma)$ and let $v_{\ell} \in C^{\infty}(\gamma)$ be a primitive of $\left(N u_{\ell}\right) \tau^{*}, 0 \leqslant \ell \leqslant N$. Assume that $h=\left(u_{\ell}+i v_{\ell}\right)_{0 \leqslant \ell \leqslant N}$ is an embedding of $\gamma$ into $\mathbb{C}^{N+1}$. Then 6.10 is a necessary condition to the existence of a Riemann surface $\mathcal{X}$ with almost smooth boundary $\gamma$ such that each $u_{\ell}$ extends to $\mathcal{X}$ as a harmonic function with harmonic conjugate function. The converse is true when $\gamma$ is connected and suitably oriented.

Remarks. Of course, the above conclusion means that $\mathcal{X}$ is a Riemann surface where each $h_{\ell}$ extends holomorphically.

Proof. If $\mathcal{X}$ exists with the required properties, $\delta=h(\gamma)$ bounds ,in the sense of current, $h(\mathcal{X}) \backslash \delta$ which is a complex curve of finite volume of $\mathbb{C}^{n} \backslash \delta$. Cauchy theorem implies then that (6.10) is verified. If (6.10) is satisfied, [18] produces a holomorphic 1-chain $Y$ such that $d Y=[\delta]$; since $\gamma$ is connected, $Y=[\mathcal{Y}]$ for a suitable orientation of $\gamma$. A normalization of $\overline{\mathcal{Y}}$ constructed as in the proof of th. 3a gives a suitable $\mathcal{X}$.

\section{REFERENCES}

[1] Ahlfors L.V. and Sario L., Riemann surfaces, Princeton Mathematical Series, No. 26, Princeton University Press, Princeton, N.J. (1960)

[2] Alexander H. and Wermer J., Linking numbers and boundaries of varieties, Ann. of Math., 151 (2000) 125-150

[3] Bossavit A., On the notion of anisotropy of constitutive laws : Some implications of the "Hodge implies metric" result, COMPEL, 20, 1 (2001) pp 233-9.

[4] Belishev M.I., Boundary control in reconstruction of manifolds and metrics (the BC method) Inverse Probl. 13, (1997) R1-R45 
[5] Belishev M.I., The Calderon problem for two dimensional manifolds by the BCmethod, SIAM J. Math. Anal. 35, no 1, (2003) 172-182

[6] Belishev M.I. and Kurylev Y.V., To the reconstruction of a Riemaniann manifold via its spectral data (BC-method) Comm. Partial Differential Equations 17 (5-6) (1992) 767-804

[7] Bishop E., Holomorphic completions, analytic continuation, and the interpolation of semi-norms, Ann. of Math. (2) 78 (1963) 468-500.

[8] Brown R. and Uhlmann G., Uniqueness in the inverse conductivity problem for non smooth conductivities in two dimensions, Comm. Part. Diff. Equations, 22 (1997) 1009-1027

[9] Calderon A.-P., On an inverse boundary problem. Seminar on Numerical Analysis and its applications to Continuum Physics (Rio de Janeiro) 65-76, Soc. Brasil. Rio de Janeiro, 1980

[10] Chirka E.M., Regularity of the boundaries of analytic sets (Russian) Mat. Sb. (N.S.) 117 (159) (1982), no. 3, 291-336, 431.

[11] Dinh T.-C., Orthogonal measures on the boundary of a Riemann surface and polynomial hull of compacts of finite length, J. Funct. Anal., 157 (1998, 624-649

[12] Dolbeault P. and Henkin G., Chaînes holomorphes de bord donné dans $\mathbb{C P}_{n}$, Bull. Soc. math. France. 125 (1997) 383-445

[13] Dolbeault P. and Poly J.-B., Variations sur le problème du bord dans $\mathbb{C P}_{n}$, preprint (1995)

[14] Federer H., Geometric measure theory, Die Grundlehren der mathematischen Wissenschaften, Band 153, Springer-Verlag New York Inc., 1969

[15] Griffiths P., Variations on a theorem of Abel, Invent. Math., 35 (1976) 321-390

[16] Griffiths, P. and Harris J., Principles of algebraic geometry, Pure and Applied Mathematics, Wiley-Interscience, New York, 1978.

[17] Harvey R., Holomorphic chains and their boundaries, Proc. Symp. Pure Math. 30 (1977) 309-382

[18] Harvey, F. R. and Lawson, H. B, Boundaries of complex analytic varieties, Ann. of Math. 102 (1975) 233-290

[19] Harvey, F. R. and Lawson, H. B, Boundaries of varieties in projective manifolds, J. Geom. Anal. 14 (2004), no. 4, 673-95

[20] Henkin G., The Abel-Radon transform and several complex variables, Ann. of Math. Stud., Princeton Univ. Press, Princeton, NJ, 1995, 223-275

[21] Lassas M. and Uhlmann G, On Determining a Riemannian Manifold from the Dirichlet-to-Neumann Map, Ann. Sci. École Norm. Sup. 34 (2001) 771-787

[22] Mandache N., Exponential instability in an inverse problem for the Schrödinger equation, Inverse Problems, 17 (2001) 1435-1444

[23] McShane E.-J., Extension of range of functions, Bull. Am. Math. Soc. 40 (1934) $837-842$

[24] Novikov R., Multidimensional inverse spectral problem for the equation $-\Delta \psi+$ $(v-E u) \psi=0$, Funct. Anal. and Appl., 22 (1988) 263-272

[25] Rosenlicht M., Generalized Jacobian varieties, Ann. of Math. (2) 59 (1954) 505-530

[26] Stolzenberg G., Volumes, limits and extensions of analytic varieties, Springer-Verlag, N.-Y., (1966)

[27] Sylvester J, An anisotropic inverse boundary value problem, Comm. Pure Appl. Math., 43 (1990) 201-232.

[28] Vekua I.N., Generalized analytic functions, Pergamon Press (1962).

[29] Wermer J, The hull of a curve in $\mathbb{C}^{n}$, Ann. of Math. 68 no 3 (1958) 550-561

(G. Henkin and V. Michel) Université Pierre et Marie Curie, 4, Place Jussieu, 75252 PARIS CEDEX 05

E-mail address: henkin@math.jussieu.fr, michel@math.jussieu.fr 